\title{
Review of structured light in diffuse optical imaging
}

Joseph P. Angelo

Sez-Jade Chen

Marien Ochoa

Ulas Sunar

Sylvain Gioux

Xavier Intes 


\title{
Review of structured light in diffuse optical imaging
}

\author{
Joseph P. Angelo, ${ }^{\mathrm{a}, *,+}$ Sez-Jade Chen, ${ }^{\mathrm{b}, *, \dagger}$ Marien Ochoa, ${ }^{\mathrm{b}, \dagger}$ Ulas Sunar, ${ }^{\mathrm{c}}$ Sylvain Gioux, ${ }^{\mathrm{d}}$ and Xavier Intes ${ }^{\mathrm{b}}$ \\ ${ }^{a}$ National Institute of Standards and Technology, Sensor Science Division, Gaithersburg, Maryland, United States \\ ${ }^{\mathrm{b}}$ Rensselaer Polytechnic Institute, Department of Biomedical Engineering, Troy, New York, United States \\ 'Wright State University, Department of Biomedical Industrial and Human Factor Engineering, Dayton, Ohio, United States \\ dUniversity of Strasbourg, ICube Laboratory, Strasbourg, France
}

\begin{abstract}
Diffuse optical imaging probes deep living tissue enabling structural, functional, metabolic, and molecular imaging. Recently, due to the availability of spatial light modulators, wide-field quantitative diffuse optical techniques have been implemented, which benefit greatly from structured light methodologies. Such implementations facilitate the quantification and characterization of depth-resolved optical and physiological properties of thick and deep tissue at fast acquisition speeds. We summarize the current state of work and applications in the three main techniques leveraging structured light: spatial frequency-domain imaging, optical tomography, and single-pixel imaging. The theory, measurement, and analysis of spatial frequency-domain imaging are described. Then, advanced theories, processing, and imaging systems are summarized. Preclinical and clinical applications on physiological measurements for guidance and diagnosis are summarized. General theory and method development of tomographic approaches as well as applications including fluorescence molecular tomography are introduced. Lastly, recent developments of single-pixel imaging methodologies and applications are reviewed. () The Authors. Published by SPIE under a Creative Commons Attribution 3.0 Unported License. Distribution or reproduction of this work in whole or in part requires full attribution of the original publication, including its DOI. [DOI: 10.1117/1.JBO.24.7.071602]
\end{abstract}

Keywords: compressed sensing; diffusion; imaging; review; spatial frequencies; tomography.

Paper 180207SSVR received Apr. 6, 2018; accepted for publication May 31, 2018; published online Sep. 14, 2018.

\section{Introduction}

The ability to probe deep tissues with light for biomedical applications has been recognized since the scientific reports published as early as the late 1800 s for monitoring brain hemorrhage $^{1}$ and early 1900s for imaging breast cancer ${ }^{2}$ and performing tissue oximetry. ${ }^{3,4}$ Since then, diffuse optical imaging techniques have greatly benefited numerous biomedical fields. Mainly, the goal of these optical techniques is to characterize optical properties of tissue using light from the ultraviolet to the infrared spectral region. The specific light-tissue interaction enables monitoring of a vast array of tissue characteristics, including structural, physiological, metabolic, and molecular properties.

The early implementations of diffuse optics were performed using wide-field sources that illuminated large areas of tissues. Such implementations enabled fast and noncontact instruments for ease of use in clinical settings. ${ }^{5,6}$ However, the collected signal is heavily surface-weighted and contrast in the biomarkers of interests are blurred due to high scattering in biotissues. ${ }^{7}$ Such inherent effects led to an undesirable number of false-positive classifications in breast cancer detection. ${ }^{8,9}$

In parallel, great strides were made to derive more accurate and efficient light propagation models. ${ }^{10,11}$ Coupled with the wide spread use of personal computers, such progress led to the development/establishment of quantitative methodologies such as functional near-infrared spectroscopy ${ }^{12,13}$ and diffuse optical tomography (DOT). ${ }^{14}$ However, such approaches were mainly based on point source and detector configurations.

*Address all correspondence to: Joseph P. Angelo, E-mail: joseph.angelo@ nist .gov; Sez-Jade Chen, E-mail: chens18@rpi.edu

TThese authors contributed equally to this work
Thus, they were not amenable to imaging large surfaces with high density source-detector configurations. Typically, they relied on a few fibers coupled to the tissue of interest, which led to sampling errors, difficulty in scaling, and high sensitivity to the optode-tissue coupling efficiency. ${ }^{15}$ Due to the recent advent of major developments in spatial light modulators (SLMs), it is now possible to combine both wide-field imaging and quantitative methodologies based on efficient light propagation models to probe large tissue surfaces and retain sensitivity to deep tissues. In this review, we summarize the current progresses in harnessing structured light strategies in diffuse optical imaging. The review covers the three main applications that have shown fast growth over the recent years: spatial frequency-domain imaging, tomography, and single-pixel imaging. Each section will outline the context and founding principles of its technology, followed by advanced methods, current limitations, and applications. The review will be concluded with an outlook of these approaches.

\section{Spatial Frequency-Domain Imaging}

Quantitative sensing of deep tissues or tomography has been largely based on point sources over the last three decades. ${ }^{16-18}$ Wilson was the first to use full-field structured illumination as a measurement tool, noting that the defocus or blurring of the structure could be used in microscopy for optical sectioning. ${ }^{19}$ In the diffuse regime, Dögnitz and Wagnières ${ }^{20}$ demonstrated that this blurring of structured illumination could be processed to a single value and analyzed as a spatial frequency response that is characteristic of the medium's optical properties. Widefield mapping $(10 \mathrm{~cm} \times 10 \mathrm{~cm})$ of diffuse optical properties was realized by Cuccia et al. ${ }^{21}$ with simple fringe pattern illumination, spatial frequency-domain measurement and analysis, and 
pixel-by-pixel processing to introduce a method termed spatial frequency-domain imaging (SFDI).

The incorporation of readily available wide-field optical components, the ease of processing in the frequency domain (as opposed to deconvolution in the spatial domain), and the quantitative nature of SFDI has made it a technology worth mentioning - as of this article, Google Scholar shows over 300 citations of the Cuccia et al. 2009 paper and Web of Science shows over 150 publications and over 800 citations for SFDI since 2010.

\subsection{Introductory Theory, Measurement, and Analysis}

\subsubsection{Theory}

In order to quantitatively map the multiple-scattered photons, i.e., the diffuse reflectance, to tissue optical parameters, a model system must be introduced. The original formulation assumes a homogeneous, linear, time-invariant system. The diffuse reflectance as a function of spatial frequency can be described with a modulation transfer function (MTF) that is characteristic of the sample's optical properties. From the perspective of signal processing, the highly scattering nature of tissue acts as a low-pass filter. The experimental and theoretical results demonstrate the loss in signal and the shortening of reflected photon pathlengths with increased spatial frequency. This means that the illumination spatial frequency dictates the optical length scale being probed. This mechanism enables depth sensing and quantification techniques. ${ }^{21,22}$

The first complete measurement and analysis for wide-field mapping of optical properties in the spatial frequency domain was done by Cuccia et al. ${ }^{21}$ and is still the foundation for most SFDI techniques. A brief walkthrough of the conceptual framework will be reiterated here-a thorough description can be found in Ref. 22. It should be noted that the framework is expounded in the diffusion regime for clarity in this context but is not limited therein.

Starting with the time-independent diffusion equation for a homogeneous medium

$$
\nabla^{2} \Phi-\mu_{\mathrm{eff}}^{2} \Phi=-3 \mu_{t r} q
$$

gives the relationship between source $q$, medium properties $\mu_{t r}=\left(\mu_{a}+\mu_{s}^{\prime}\right) \quad$ (transport coefficient with absorption and reduced scattering coefficients $\mu_{a}$ and $\mu_{s}^{\prime}$, respectively) and $\mu_{\text {eff }}=\left(3 \mu_{a} \mu_{t r}\right)^{1 / 2}$, and fluence rate $\Phi$. Assuming a semi-infinite geometry, we now introduce a pure spatially modulating source that is periodic and normally incident to the boundary surface

$q=q_{0}(z) \cos \left(k_{x} x+\varphi\right)$,

which modulates in the $x$-spatial dimension with spatial frequency $f_{x}=k_{x} / 2 \pi$ and phase $\varphi$ and is constant across the $y$-spatial dimension with arbitrary depth dependence $q_{0}(z)$. Assuming a linear medium, this modulated source will result in a modulated fluence rate with the same frequency and phase

$\Phi=\Phi_{0}(z) \cos \left(k_{x} x+\alpha\right)$.

Modulation is allowed for an arbitrary direction and the $y$-spatial dimension is held constant here for convenience of analysis. These equations are combined to form a one-dimensional (1-D) second-order Helmholtz equation $\frac{d^{2}}{d z^{2}} \Phi_{0}(z)-\mu_{\mathrm{eff}}^{\prime 2} \Phi_{0}(z)=-3 \mu_{t r} q_{0}(z)$,

where

$\mu_{\mathrm{eff}}^{\prime}=\left(\mu_{\mathrm{eff}}^{2}+k_{x}^{2}\right)^{1 / 2} \equiv \frac{1}{\delta_{\mathrm{eff}}}$.

Here, $\delta_{\text {eff }}^{\prime}$ defines the effective penetration depth, a useful construct for estimating and comparing the depth sensitivity in the spatial frequency domain to other domains. First, note that with $k_{x}=0$ the penetration depth is simply $1 / \mu_{\text {eff }}$, as expected from a constant, planar illumination source. ${ }^{23}$ Next, the penetration depth decreases with increasing spatial frequency-a key result that enables depth sensing and tomography (see Secs. 2.2.1 and 3). ${ }^{21}$ Equation (4) is identical to the diffusion equation for continuous planar illumination, and so previous solutions can be utilized with partial-current boundary conditions $^{24}$ to yield the following expression for the diffuse reflectance:

$R_{d}\left(k_{x}\right)=\frac{3 A \mu_{s}^{\prime} / \mu_{t r}}{\left(\frac{\mu_{\mathrm{eff}}^{\prime}}{\mu_{t r}}+1\right)\left(\frac{\mu_{\mathrm{eff}}^{\prime}}{\mu_{t r}}+3 A\right)}$,

where

$A=\frac{1-R_{\mathrm{eff}}}{2\left(1+R_{\mathrm{eff}}\right)}$

and

$R_{\mathrm{eff}} \approx 0.0636 n+0.668+\frac{0.710}{n}-\frac{1.440}{n^{2}}$,

where $A$ is a proportionality constant formed from the effective reflection coefficient $R_{\text {eff }}$, obtained by integrating the Fresnel reflection coefficient over all angles of incidence for unpolarized light, and index of refraction ratio $n$, generalized as a bulk property.

The diffuse reflectance $R_{d}\left(k_{x}\right)$ is effectively the spatial modulation transfer function of the medium, i.e., the spatial frequency response function of the linear system. As demonstrated in other domains, the diffuse nature of tissue characterizes its response as a low-pass filter in the spatial frequency domain. The diffusion approximation remains valid when $\mu_{s}^{\prime} \gg \mu_{a}$ and when the spatial frequency $f_{x}$ illuminating the sample is much less than the transport coefficient $\mu_{t r}$. For tissue measurements, in practice this means the maximum spatial frequency expected to satisfy the diffusion model is $\sim 0.33 \cdot \mu_{t r} \cdot{ }^{22} \mathrm{New}$ analytical models have been introduced to characterize the diffuse reflectance beyond the diffusion regime where photons are minimally scattered due to increased absorption or shorter photon pathlengths. ${ }^{25,26}$

\subsubsection{Measurement}

Equation (2) depicts a pure sinusoidal source illuminating the sample, but in practice this is impossible-one cannot illuminate with negative intensity - and so a DC offset is necessary to support the AC carrier frequency of source $S$ along the $x$ dimension

$S=\frac{S_{0}}{2}\left[1+M_{0} \cos \left(2 \pi f_{x} x+\phi\right)\right]$ 


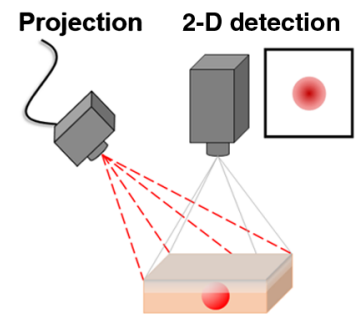

(a)

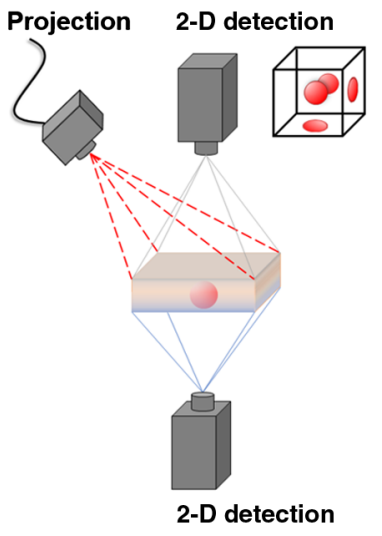

(b)

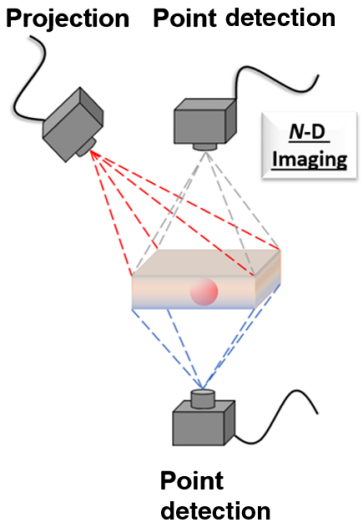

(c)

Fig. 1 Schematics of typical systems for (a) SFDI, (b) tomography, and (c) single-pixel imaging. Dotted lines indicate structured projection or detection, usually by DMD, and solid lines indicate 2-D camera detection. (a) SFDI offers a simple model, rapid collection, and depth-averaged imaging. (b) Tomography can be in reflection (gray) or transmission (blue) geometry, requires advanced models, processing, and many acquisitions for 3-D reconstructions. (c) Single-pixel detection is highly flexible in its geometry, detection (temporal, frequency, spectral, and/or spatial resolution), for $N-D$ imaging.

where $S_{0}$ is the source intensity and $M_{0}$ is the modulation depth (set to 1 for maximum AC signal). One can see that with a simple 1-D sinusoidal projection, the sample will simultaneously be illuminated with a $S_{0} / 2$ DC signal with no modulation and a $S_{0} / 2\left[M_{0} \cos \left(2 \pi f_{x} x+\phi\right)\right]$ AC wave modulating at $f_{x}$. It is worth noting that this simultaneous $\mathrm{AC}$ and DC sampling has been utilized for advanced acquisition methods (see Sec. 2.2.2). For now, consider that the reflected AC intensity $I_{\mathrm{AC}}$ incorporates the tissue's response in the amplitude envelope $M_{\text {AC }}$ seen here

$I_{\mathrm{AC}}=M_{\mathrm{AC}}\left(x, f_{x}\right) \cos \left(2 \pi f_{x} x+\phi\right)$.

To retrieve a single measurement $M_{\mathrm{AC}}$ for a given frequency, the most widely used method is the signal processing technique of phase stepping, where three images are collected $I_{1}, I_{2}, I_{3}$, one at each step of $\phi=[0,120 \mathrm{deg}, 240 \mathrm{deg}]$. This procedure is explained for multiple modalities by Mertz. ${ }^{27}$ These images are processed together for demodulation, i.e., to remove the carrier frequency along with the DC signal and to obtain the amplitude envelope at each image pixel $x_{i}$

$$
\begin{aligned}
M_{\mathrm{AC}}\left(\mathrm{x}_{i}, f_{x}\right)= & \frac{2^{1 / 2}}{3}\left\{\left[I_{1}\left(x_{i}\right)-I_{2}\left(x_{i}\right)\right]^{2}+\left[I_{2}\left(x_{i}\right)-I_{3}\left(x_{i}\right)\right]^{2}\right. \\
& \left.+\left[I_{3}\left(x_{i}\right)-I_{1}\left(x_{i}\right)\right]^{2}\right\}^{1 / 2}
\end{aligned}
$$

This amplitude envelope $M_{\mathrm{AC}}$ is the product of the initial source intensity $I_{0}$, the optical system's modulation transfer function $\mathrm{MTF}_{\text {sys }}$, and the sample's response contained in the diffuse reflectance $R_{d}\left(x_{i}, f_{x}\right)$

$M_{\mathrm{AC}}\left(x_{i}, f_{x}\right)=I_{0} \mathrm{MTF}_{\mathrm{sys}} R_{d}\left(x_{i}, f_{x}\right)$.

Because the measurement and analysis is done in the spatial frequency domain, $M_{\mathrm{AC}}$ is in fact the product of the response functions, allowing for a simple ratio against a reference sample of known optical properties to remove the systematic components and retrieve the sample's diffuse reflectance ${ }^{22}$
$R_{d}\left(x_{i}, f_{x}\right)=\frac{M_{\mathrm{AC}}\left(\mathrm{x}_{i}, f_{x}\right)}{M_{\mathrm{AC}, \text { ref }}\left(\mathrm{x}_{i}, f_{x}\right)} R_{d, \text { ref,pred }}\left(f_{x}\right)$.

A light propagation model is used to scale the referenced measurement by $R_{d \text {,ref,pred }}\left(f_{x}\right)$ and an inverse solver is then used to retrieve optical properties from sample diffuse reflectance measurements. As few as two spatial frequency measurements, one $\mathrm{AC}$ and one $\mathrm{DC}\left(f_{x}=0\right)$, can be used to properly generate absorption and reduced scattering maps. ${ }^{22}$

\subsubsection{Analysis}

The above measurement is analyzed pixel-by-pixel, resulting in a wide-field map of optical properties. Because each pixel is processed independently, technology suited for wide-field use can be readily implemented. System components vary with application, but the most common implementations utilize a digital micromirror device (DMD) for projection and a charged couple device (CCD) or a complementary metal-oxide-semiconductor (CMOS) camera for imaging [see Fig. 1(a)].

To achieve optical property maps of the measured sample, an inverse solver is required for mapping $R_{d}\left(x_{i}, f_{x}\right)$ to $\mu_{a}$ and $\mu_{s}^{\prime}$. While diffusion theory [see Eq. (6)] might be preferred as the forward model and inverse solver due to its speed and ease of use, Monte Carlo models and empirical look-up tables can be precalculated for rapid solutions beyond the diffusion regime. $^{22,28-30}$ While simulations in the spatial frequency domain can be done with a complex weighting scheme, ${ }^{29}$ simulations are often done in the spatial domain as a point source and then transformed to the spatial frequency domain, e.g., by radially symmetric 1-D Hankel transform ${ }^{22}$ or by convolution to a line source and a 1-D Fourier transform. ${ }^{31}$

Early analysis methods demonstrated the depth-sensitivity dependence on spatial frequency in diffusive media ${ }^{21,32}$ and its potential for increased contrast with fluorescent inclusions. ${ }^{33}$ Single wavelength analysis was expanded to multispectral measurements, enabling quantitative chromophore concentration measurements ${ }^{34}$ and mapping of physiological markers with as few as two wavelengths. ${ }^{35}$ To extend SFDI's clinical relevance, a profile-correction scheme was developed so nonflat samples 


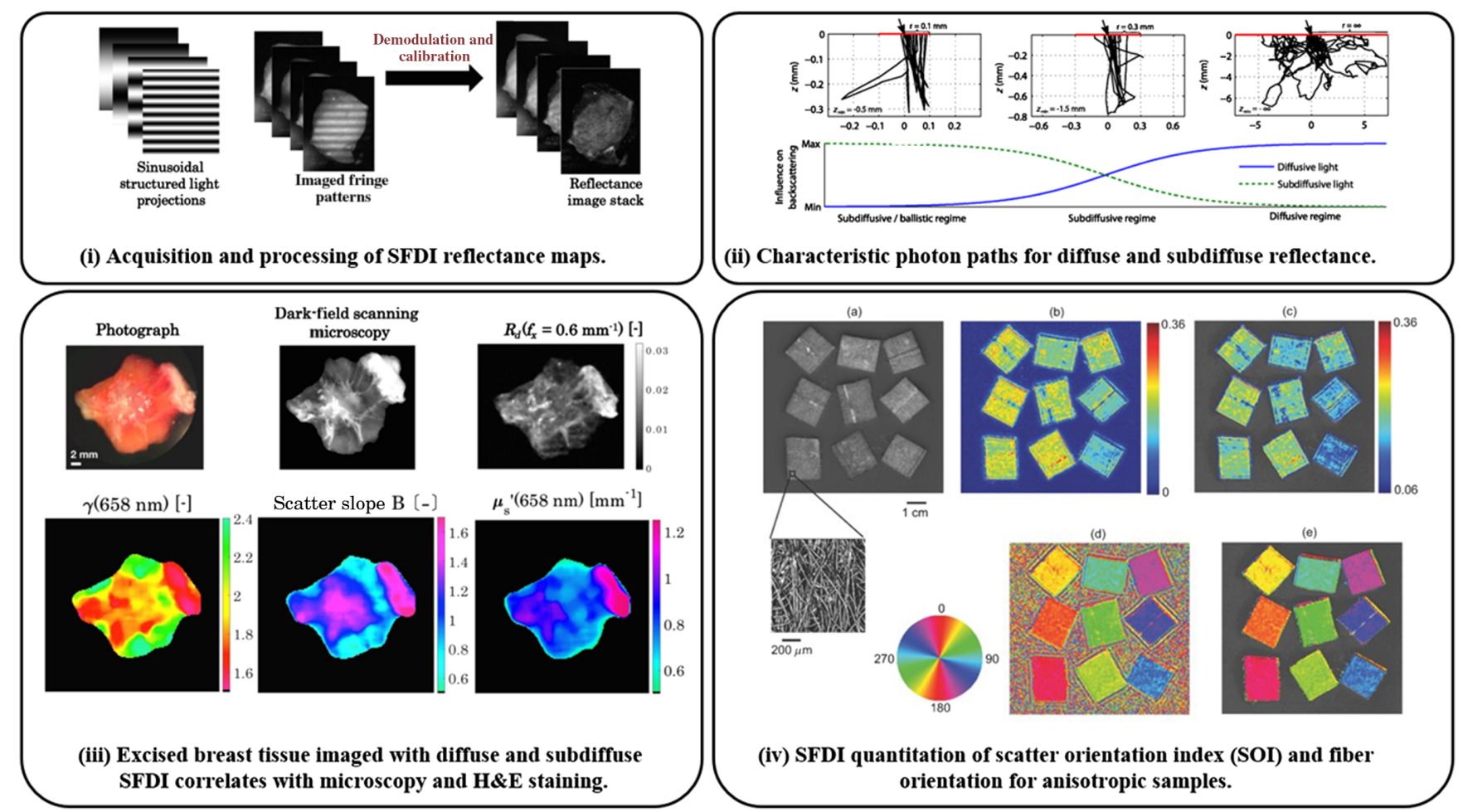

Fig. 2 Acquisition, modeling, and application: (i) demonstration of structured illumination patterns, collection, and demodulated reflectance images-adapted from Ref. 45. (ii) Average photon paths get shorter and less diffuse with decreased source-detector separation, similar to increasing spatial frequency-adapted from Ref. 47. (iii) Subdiffuse SFDI demonstrates its ability to measure scatteringrelated parameters that correlate with histology of excised cancerous breast tissue-adapted from Ref. 45. (iv) SFDI demonstrates sensitivity to the amount of anisotropy (top row) and fiber orientation (bottom row)-adapted from Ref. 48.

could be measured. ${ }^{36}$ Acquisition could be relatively fast (on the order of seconds), but this is too slow to avoid certain motion artifacts such as breathing. Therefore, a correction scheme was devised to correct for these artifacts, ${ }^{37}$ though current methods focus instead on rapid acquisition to avoid the issue (see Sec. 2.2.2). ${ }^{38-40}$

\subsection{Advanced Techniques}

From the fundamentals discussed in the first section, research has expanded the theory, processing, instrumentation, and modeling to develop additional robust techniques that push the limits of quantitative imaging technology.

\subsubsection{Advanced theory}

Using the diffusion theory result for reflectance [see Eq. (6)], one can derive that with planar illumination $\left(k_{x}=0\right)$ absorption has its maximum effect on the diffuse reflectance $R_{d}\left(k_{x}\right){ }^{22}$ Furthermore, the diffuse reflectance sensitivity to multiple light scattering $\left(\mu_{s}^{\prime}\right)$ is also maximum in this regime, but increasing spatial frequency, particularly passed the diffuse regime $\left(k_{x} \gg \mu_{\text {eff }}\right)$, decreases multiple light scattering and increases sensitivity to the scattering phase function. Enabling both diffuse and subdiffuse imaging into a single modality has further demonstrated the utility of structured illumination for imaging biological tissue.

Entering the subdiffuse regime [cf. Fig. 2(ii)], studies have shown that with increasing spatial frequency, the reflectance signal becomes increasingly sensitive to the medium's scattering phase function $P\left(\theta_{s}\right){ }^{26,41}$ Sensitivity beyond the scattering phase function's first Legendre moment, anisotropy factor $g_{1}=\cos \left(\theta_{s}\right)$, is often characterized by combining the first two Legendre moments to form scattering parameter $\gamma=\frac{1-g_{2}}{1-g_{1}}{ }^{4}, 43$ With this added phase function sensitivity, studies have demonstrated the characterization of the fractal size distribution of Mie scatterers in phantoms ${ }^{26,44}$ and McClatchy et al. ${ }^{45,46}$ even classified tumor grades in ex vivo human breast and murine cancer models as compared to histopathology [see Fig. 2(iii)].

However, with the new rapid modeling capabilities of their analytical radiative transport equation (RTE) solution, ${ }^{25,49}$ Liemert et al. ${ }^{47}$ showed that $\gamma$ may be prone to errors due to underestimating the number of high-angle scattering events for high spatial frequency reflectance measurements. As a solution, they proposed to weight the higher order Legendre moments of the phase function $P\left(\theta_{s}\right)$ with a shaping factor $c$

$$
\sum_{i=2}^{\infty}(-c)^{i-2} \frac{1-g_{i}}{1-g_{1}}=\gamma-c \delta+c^{2} \varepsilon-c^{3} \zeta+\ldots-\ldots
$$

that determines the decreasing weight of the higher order parameters $\gamma, \varepsilon, \zeta$, etc. After extensive modeling for various scattering phase functions and collection geometries, the best average value was $c=0.5$. This is used to define their scattering parameter $\sigma$

$\sigma=\sum_{i=2}^{\infty}(-0.5)^{2} \frac{1-g_{i}}{1-g_{1}}$ 
that outperforms $\gamma$ in precision. ${ }^{47}$ However, the only testing of $\sigma$ thus far has been with simulation, and there is extensive literature for comparison of $\gamma$ measurements in other domains. ${ }^{42,43,50-52}$

Stepping up in length scales from the scattering phase function, wide-field optical imaging techniques generally have poor sensitivity to microscopic scattering structures, such as fiber orientation. However, Konecky et al. ${ }^{48}$ developed an anisotropic diffusion model to simulate and experimentally validate the sensitivity of structured illumination to fiber orientation [cf. Fig. 2(iv)]. By analyzing the amplitude and phase shift of the structured illumination, Konecky et al. deduced the fiber orientations as well as assessed the strength of anisotropy in a scattering orientation index (SOI) defined as

$$
\mathrm{SOI}=\max _{\varphi}\left\{\frac{|g(\varphi)-| g(\varphi+\pi / 2)||}{|g(\varphi)+| g(\varphi+\pi / 2)||}\right\},
$$

where $g(\varphi)$ is the angular-dependent anisotropy factor of the medium and $\varphi$ is the angle of the projected spatial frequency.

Broader still, the original modeling of SFDI requires a homogeneous medium, but several studies have developed models and evaluated two-layer systems for a compromise between the gain in depth resolution and the cost of processing and acquisition speed. ${ }^{53-55}$ These models would go on to support studies evaluating the effects of melanin concentrations in skin on optical measurements, ${ }^{56,57}$ as well as the layering of intralipid during the phantom experiments. ${ }^{58}$ In addition to correcting for the confounding effects of melanin on chromophore reconstruction using spectroscopy, the depth resolution enables a density measure that is compared to and confirmed with nonfluorescent multiphoton microscopy measurements. ${ }^{56}$

With concern for robustness and quantitation, limitations and sources of error have also been well studied for diffuse structured illumination imaging. A thorough study by Bodenschatz et al. ${ }^{59}$ has developed a list of guidelines to minimize error and to assess the sensitivity of measurement-related parameters. In short, these guidelines state to avoid analyzing the boundary of the illumination field, eschew over-binning camera pixels (though this is minor), limit changes the camera-sample distance or use a correction scheme, ${ }^{36,39,60}$ and ensure accurate determination of the projected spatial frequency. Another confounding factor is the popular use of cross-polarization without the proper modeling of polarized photon transport. While cross-polarization is useful to remove specular reflections, this lack of consistency can lead to errors in $\mu_{a}$ of up to $25 \% .{ }^{31}$

\subsubsection{Advanced processing}

The development of SFDI had immediate appeal because of its wide-field, quantitative imaging, but SFDI was mostly utilizing tools and techniques developed for separate spatial, temporal, or frequency domains. With tools and processing built specifically for spatial frequency-domain measurements, new applications are possible and clinical relevance materializes (see Sec. 2.3).

Speed is a major concern for a clinically relevant imaging system. To map the optical properties of a given sample at a single wavelength using SFDI with phase-stepping demodulation for AC signal and DC background subtraction, at least six images need to be processed using Eq. (11) [cf. Fig. 2(i)]. Although this approach is robust, it requires a static sample or a motion-correction algorithm, ${ }^{37}$ though the latest demodulation techniques have minimized the number of required images. A simple approach to cut down the number of acquisitions is to average any three $\mathrm{AC}$ images to form the DC image for processing. Nadeau et al. ${ }^{40,61}$ reduced acquisition requirements down to two images by utilizing a two-dimensional (2-D) Hilbert transform technique. The Hilbert transform technique avoids digital DC filtering by subtracting the DC image from the AC image and seems to keep most of the original image's resolution, though there is an added complexity of rapidly producing and alternating two patterns and syncing with collection hardware. Nadeau et al. ${ }^{61}$ further advanced rapid acquisition methods by demonstrating the multifrequency capabilities of a square-wave projection. Work by Vervandier and Van de Giessen has pushed acquisition down to a single image using single snapshot of optical properties (SSOP) imaging. $38,39,62,63$ SSOP requires digital separation of $\mathrm{DC}$ and $\mathrm{AC}$ frequencies that adds complexity to keeping image resolution, though its single image acquisition allows for highly simplified pattern production and image collection. SSOP has been further extended to three-dimensional (3-D) height corrections using 3D-SSOP and has demonstrated real-time imaging of a heartbeat waveform with 50 frames per second. ${ }^{39,62}$ Another variation that involved single snapshot and multiple frequency modulation also has been proposed, where MTF with respect to spatial frequency provides quantification of absorption and scattering parameters. ${ }^{64}$

Beyond acquisition, further developments have pushed for forward and inverse model solving. Martinelli et al. ${ }^{65}$ developed scaling relationships from the RTE that enable the forward modeling of spatially and temporally resolved reflectance using a single Monte Carlo simulation. Yang and $\mathrm{Choi}^{66}$ accelerated Monte Carlo simulations using a graphical processing unit (GPU) specifically with SFDI in mind. Though memory transfer from CPU to GPU is still a limiting factor, a 3400-fold improvement was shown over other GPU-based approaches for multiple diffuse reflectance simulations. Furthermore, recent work by Pera et al. ${ }^{67}$ has demonstrated a method to provide uncertainty estimates of SFDI Monte Carlo solutions for optical properties and chromophore fitting. Based on analytical solutions to the RTE, Liemert et al. ${ }^{25,49,68}$ demonstrated several solutions for rapid, highly accurate simulations for various domains in a matter of seconds. This enables quick and extensive parameter testing for model error and measurement sensitivity. ${ }^{41,47}$ With the aim of enabling real-time processing for clinical use, Angelo et al. ${ }^{30}$ developed rapid look-up table methods that demonstrate a 100-times faster solution over previously published methods.

\subsubsection{Advanced systems}

The relatively simple and inexpensive instrumentation needed for structured illumination imaging means that expansion and development are inevitable. Demonstrating structured illumination imaging's potential for broad accessibility, groups have developed low-cost systems made from commercial components that are simplified, portable, and capable of accurate quantitative imaging. ${ }^{69,70}$ SFDI has also been expanded from the visible and near-infrared (NIR) into the short-wave infrared region by utilizing InGaAs detector arrays. ${ }^{71}$ The spectral density was also increased with work by Singh-Moon et al. ${ }^{72}$ using a line-scan hyperspectral detector. Previous work by Saager et al. ${ }^{34,57}$ demonstrated spatial frequency-domain spectroscopy point measurements, but 2-D imaging potential lies with hyperspectral point detection using compressed-sensing SFDI. ${ }^{73}$

With an even deeper focus on translation, several groups have developed systems for specific clinical problems. While 


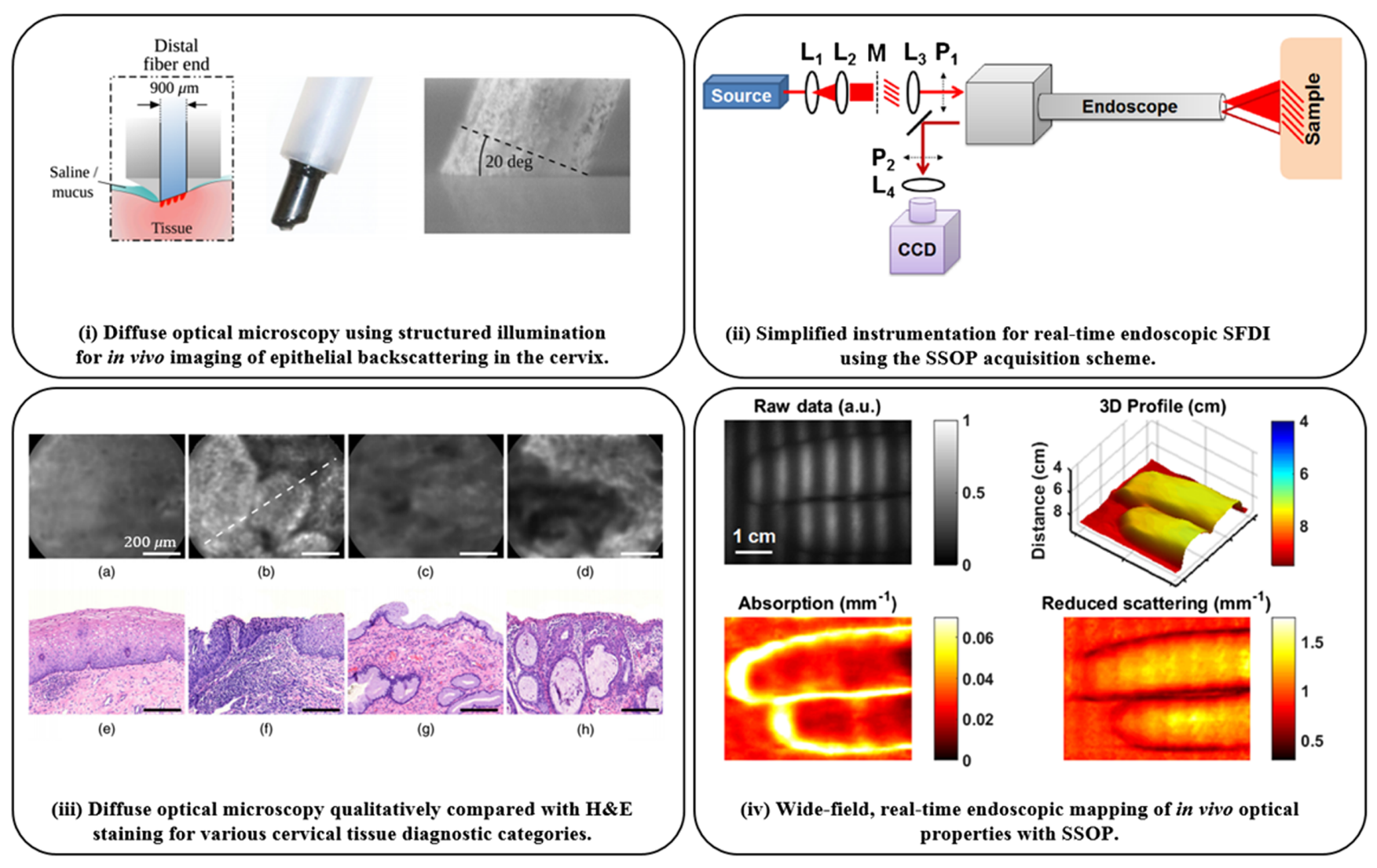

Fig. 3 Endoscopic structured illumination imaging: (i) fiber-based probe with structured illumination for diffuse optical microscopy (DOM). (ii) 3D-SSOP enables real-time, quantitative endoscopic wide-field imaging with simplified components: lenses $L$, mask $M$, and polarizers $P$. (iii) (a-d) In vivo imaging using DOM of cervical tissue (e-h) with corresponding H\&E histology- columns (a) and (c) are benign and columns (b) and (d) are precancerous. (iv) Endoscopic SSOP producing 3-D profile, absorption, and reduced scattering maps from a single raw frame, enabling video-rate imaging in vivo. (i) and (iii) are adapted from Ref. 79 and (ii) and (iv) are adapted from Ref. 81.

most are custom in-house systems, the FDA has given its first 510(k) approval to a commercial SFDI system for application in oxygenation imaging for diabetic foot ulcers. ${ }^{74-76}$ While this brings the standard wide-field system to fruition, efforts by other groups have been made to develop structured illumination endoscopic imaging systems for minimally invasive clinical guidance. ${ }^{77-80}$ Applying standard three-phase SFDI processing to a fiber-contact endoscope system enables high spatial frequency imaging $\left(f_{x}=[2.7\right.$ to 14.5$\left.] \mathrm{mm}^{-1}\right)$ for cancer detection [cf. Figs. 3(i) and 3(iii)], yet suffers the same reduced overall frame rate as wide-field processing and care must be taken to avoid motion artifacts. ${ }^{79}$ Higher frame rates are achieved with a noncontact, three-dimensional (3-D) corrected endoscopic imaging approach [shown in Figs. 3(ii) and 3(iv)] by utilizing SSOP acquisition and processing, enabling real-time imaging of optical and vascular properties for dynamic samples. ${ }^{77,78}$ With new technical developments, clinical usage and accessibility of SFDI become practical.

The simplicity of structured illumination imaging lends itself to multimodal measurements with other clinical techniques. Ghassemi et al. ${ }^{82}$ combined out-of-plane Stokes polarimetry with multispectral SFDI to study hypertrophic scars for surface roughness and pathophysiology with a single system. Though each modality has a unique illumination pathway, the shared collection pathway produces inherently coregistered measurements for direct correlation. To better aid surgery and photodynamic therapy (PDT), Rohrbach et al. ${ }^{83}$ performed a feasibility study with SFDI alongside high frequency ultrasound for the combined functional and structural information. Similarly, Lin et al. ${ }^{84}$ obtained multimodal and multiscale images using SFDI, Doppler optical coherence tomography (OCT), and confocal microscopy to study Alzheimer's disease-dependent changes in a mouse brain model. Furthermore, McClatchy et al. ${ }^{85}$ combined microcomputed tomography (micro-CT) with SFDI to potentially aid in the assessment of tumor margin status in breast conserving surgery. These works open new pathways to clinical efficacy, although much work is needed from the field before multimodal systems come to full form.

\subsection{Applications}

Advanced theory, analysis, and instrumentation have bolstered the understanding of structured illumination imaging while pushing the boundaries of which applications seemed possible.

\subsubsection{Photon gating and quantification}

The simple projection and collection scheme of structured illumination make it readily adaptable and practical. Slightly different than multimodal systems, some techniques implement the theory, analysis, or processing of structured illumination to directly augment another technique by either gating photons selectively or providing a quantitative foundation. 


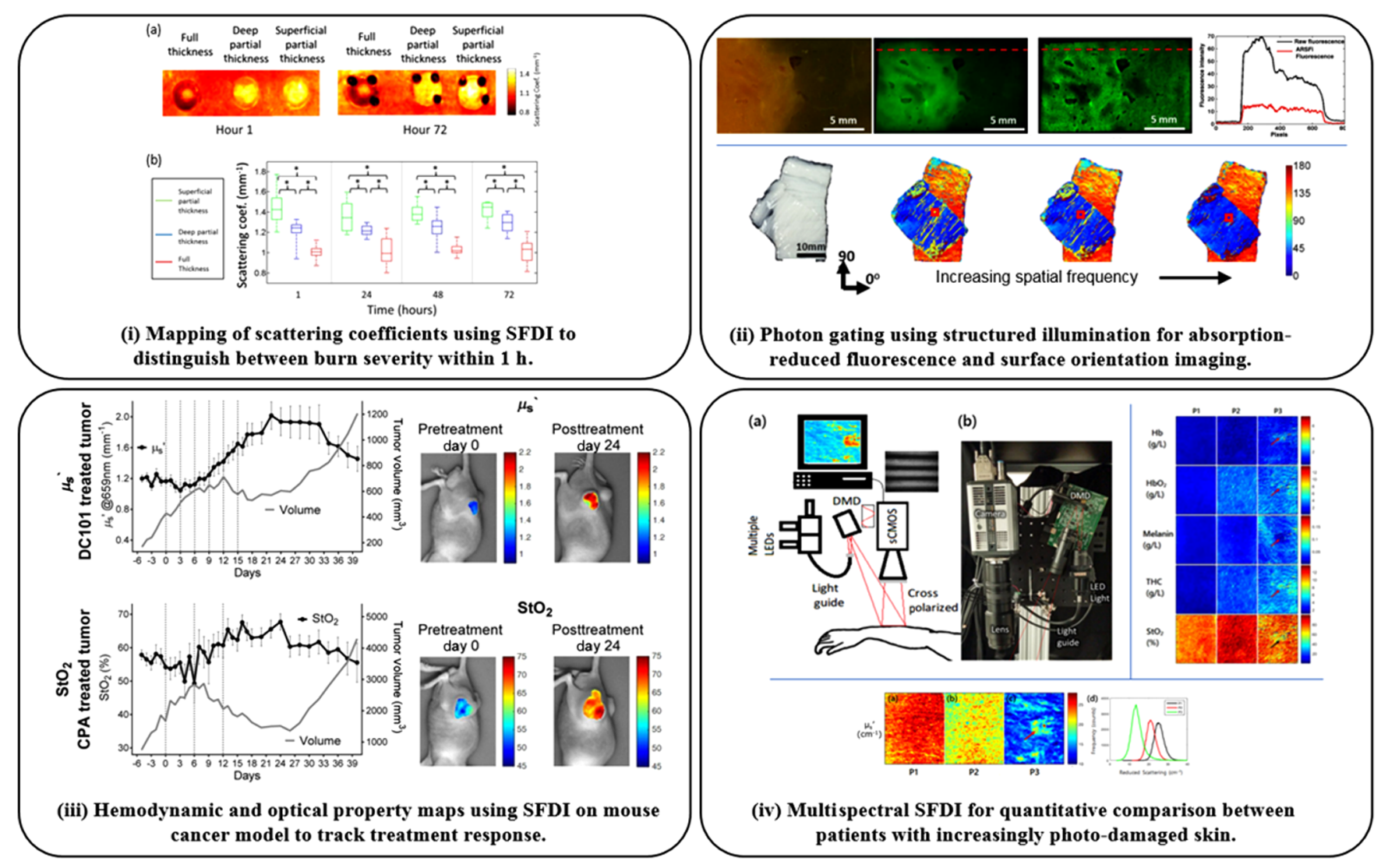

Fig. 4 Toward clinical applications using SFDI: (i) SFDI predicts burn severity in a porcine model within $1 \mathrm{~h}$ using scattering coefficient imaging - adapted from Ref. 89. (ii) Photon-gating with increased spatial frequency enables absorption-reduced fluorescence imaging (top) and surface fiber orientation imaging (bottom) -adapted from Refs. 101, 102. (iii) Preclinical longitudinal study of tumor growth and chemotherapy response demonstrate feasibility for quantitative tracking of cancer therapies with SFDIadapted from Ref. 76. (iv) Actinic skin damage assessment using mesoscopic SFDI (top-left) for mapping chromophores (top-right) and the reduced scattering coefficient (bottom) to highlight photodamage in patient P3-adapted from Ref. 116.

Because pattern projection is often done with a laser source, laser speckle is a free product that can be utilized for enhanced laser speckle imaging (LSI). ${ }^{86,87}$ The marriage of wide-field quantitative optical property measurements from SFDI and flow contrast from LSI can be combined to a quantitative, depth-resolved measure of particle flow. ${ }^{88}$ Furthermore, SSOP processing can be implemented to provide real-time quantitative flow imaging. ${ }^{62}$ With the clinic in mind, SFDI/LSI has thoroughly demonstrated potential for real-time burn assessment (see Sec. 2.3.2). ${ }^{89-91}$

Quantitation has been a long-time goal for fluorescence imaging, so the advent of a wide-field quantitation technique brought a flurry of research to merge the two. ${ }^{63,92-96}$ SFDI measurement and analysis of the sample acquire wide-field diffuse optical properties that are then used in a correction model for PDT $^{92,94}$ and surgical guidance ${ }^{63,93,95}$ to account for excitation and/or emission wavelength losses due to absorption and scattering. For SFDI to keep up with fluorescence imaging speeds, SSOP processing can enable real-time optical-property-corrected fluorescence imaging. ${ }^{63}$ While demonstrating substantial improvement over standard fluorescence imaging corrections, SFDI-corrected fluorescence imaging has depth, solvent, and $\mathrm{pH}$ conditions that potentially confound the precise quantification of fluorophore concentration, though work has demonstrated that $\mathrm{pH}$ quantitation is possible through quantum yield mapping. ${ }^{96}$
Much like polarization, structured illumination can be used as a gating tool to preferentially filter photons by pathlength. ${ }^{97}$ Imaging with increased spatial frequency decreases the average collected photon path length, resulting in a decreased average photon penetration depth ${ }^{21,22,33}$ and increased sensitivity to shorter path length interactions. ${ }^{41,98}$ The spatial pattern can be adjusted during fluorescence imaging to vary the depth and contrast, ${ }^{33,99,100}$ or pushed high such that sensitivity to absorption is lost [cf. Fig. 4(ii), top]. ${ }^{101}$ This loss of absorption sensitivity comes with added sensitivity to scattering structures and can be utilized for highlighting scattering anisotropies due to fibrous tissue $^{102}$ [cf. Fig. 4(ii), bottom] or skin pathologies. ${ }^{98,103}$ The benefit of gating with structured light is that it can be dynamically adjusted to suit the depth and contrast needed for the sample's optical properties. This potentially enables any planar illumination, wide-field imaging technique to capture subdiffuse contrast.

\subsubsection{Physiological measurement for guidance and diagnosis}

Applying spatial frequency-domain analysis to structured illumination measurements, especially over several wavelengths, provides quantitative ground for further analysis. Some physiological correlations are made directly from optical properties, 
whereas some techniques perform spectroscopy to recover vascular parameters, and others use fluorescence for guidance and diagnosis.

A first-in-human pilot study for clinical SFDI used during breast reconstructive surgery was performed in $2010 .^{104}$ This feasibility study presented SFDI's capability for performing oxygenation imaging during a skin flap transplant procedure. Similar models have since been studied in animals to validate the utility of SFDI evaluation of flap profusion and viability. ${ }^{105-107}$ While skin flap viability is classically assessed by visual inspection, SFDI has shown promise in detecting profusion changes before they are perceptible to the eye by tracking vascular parameters such as total hemoglobin concentration and oxygen saturation. ${ }^{108}$

The functional and structural changes associated with burn wounds, along with their clinically difficult assessment, make the endogenous contrast and noncontact measurement of structured illumination an attractive choice as a monitoring tool. Work has largely focused on the ability of SFDI to predict and distinguish burn wound severity in rat and pig models, demonstrating that vascular parameters can distinguish between partial-superficial and full thickness burns after 1 day ${ }^{109}$ and the structural parameter, i.e., the reduced scattering coefficient, is able to separate all second degree burn severities within $1 \mathrm{~h}$ [cf. Fig. 4(i)]..$^{89,110}$ While histopathology is the gold standard for evaluating burn depth, it is often avoided due to its invasiveness. However, a recent study successfully correlated the invasive histopathology of a porcine burn model with the noninvasive combined sensitivity of SFDI and LSI to corroborate their potential for clinical use. ${ }^{90}$ Similarly, other combinations for multimodal systems, e.g., polarimetry with spectral SFDI ${ }^{82}$ and laser Doppler imaging with SFDI, ${ }^{111}$ have been used to investigate burn wound infections and scar formation with noncontact endogenous imaging.

Brain imaging with structured illumination has shown potential for several applications. Alzheimer's disease in mouse models can be detected with the functional imaging of SFDI, ${ }^{84,112}$ and the corresponding neuronal cell death correlates to its scattering parameter. ${ }^{113}$ A major push for quantitative-fluorescence imaging is for tumor targeting, particularly for precious tissue such as the brain. As demonstrated by Konecky et al. ${ }^{114}$ and Sibai et al., ${ }^{115}$ SFDI can be used to quantify the fluorophore concentrations in a wide-field mapping for glioma resection. In addition, brain imaging with hyperspectral SFDI was correlated with point-based optical pharmacokinetics measurements to successfully trace drug delivery concentrations to glioma tumor sites. ${ }^{72}$

The spatial heterogeneity of cancer growth and the associated high rates of secondary excisions make the spatial mapping and quantitative sensing of structured illumination a potentially powerful clinical aid. In a broad view, SFDI has been used in preclinical models to monitor the efficacy of cancer therapies to aid diffuse optical methods in the clinic [cf. Fig. 4(iii)] ${ }^{76}$ Aimed at direct clinical intervention, several studies have demonstrated the potential of structured illumination for specific tissue-type diagnosis in ex vivo tissue, such as breast tissue (diffuse $^{74,117}$ and subdiffuse ${ }^{45}$ ) and ovarian tissue. ${ }^{118}$ Both the functional and structural properties measured with structured illumination help quantify tissue viability. ${ }^{46,74}$ Furthermore, the pilot study for a nonmelanoma skin cancer clinical trial with SFDI optical and vascular parameters presented a clear separation of healthy tissue and each lesion stage of precancerous actinic keratosis as seen in Fig. 4(iv). ${ }^{116}$

\section{Tomography}

\subsection{Introduction and General Theory}

Fast and quantitative mapping of optical contrasts over large surfaces benefits numerous biomedical applications, but the fundamentals of light-matter interaction typically limit the use of single-projection imaging techniques to the superficial sensing of tissues without the ability to retrieve depth information and 3-D shapes. When deeply embedded contrasts are targeted and 3 -D volumetric imaging is required, more sophisticated methods that involve an inverse optical problem are employed and are broadly labeled optical tomography. Optical tomography can be performed at multiple length scales ranging from optical projection tomography, which focuses on millimeter-scale transparent specimens, ${ }^{119}$ to mesoscopic fluorescence molecular tomography (FMT) and macroscopic DOT, both of which harness scattering photons to retrieve the biodistribution of biomarkers of interest a few millimeters deep or over centimeter scales, respectively. Herein, we will focus on macroscopic DOT and the readers interested in the mesoscopic regime can refer to Ref. 120.

Macroscopic optical tomography principles were developed in the late 1980s and early 1990s with focus on retrieving absorptive inhomogeneities deep within a sample. Over the years, the technique(s) has been improved to image multiple endogenous biomarkers and termed DOT. In addition, the principles of DOT have been adapted to image fluorescence signals and for fluorescence applications, termed FMT. ${ }^{121-125}$ In both cases, traditional macroscopic tomography systems were designed around multiple point sources or detectors that were raster-scanned or consisted of large fiber bundles, and some systems contained a stage for rotation of the sample or the source and detector planes. ${ }^{126}$ Eventually, many researchers adopted CCD cameras as the detection scheme for parallel acquisition and illumination. DOT can be performed efficiently using continuous wave $(\mathrm{CW})^{127,128}$ systems with the advantage of robustness and ease of implementation. More challenging are frequency-domain ${ }^{126,129,130}$ and time-domain instrumental implementations. ${ }^{125,131,132}$ However, these implementations enable the measurement of time-dependent data sets such as modulated amplitude and phase, ${ }^{14,133}$ time gates, ${ }^{134}$ or transformed data $^{135}$ with the benefit of higher information content for more accurate optical tomographic reconstructions.

To perform optical tomography, the volume to be imaged is discretized in elements of volume and the inverse problem aims at retrieving the value of the biomarker of interest in each of these volume elements (or $x$ below). To do so, a photon propagation model of the light fluence is generated in order to calculate the contribution, or "weight," of each volume element in the sample to the overall measurements. Then, a classical inverse problem is formulated, i.e., $A x=b$, where $A$ is the Jacobian (or sensitivity) matrix generated from the forward model and $b$ is the measured data, and then solved to determine the values of $x$ over the whole volume. The main principles for reconstruction have not altered significantly over time, but due to developments in computational power, there have been great improvements in the efficiency and accuracy of reconstruction algorithms. For simple geometries, researchers have generated the sensitivity matrix analytically, but higher-complexity samples require numerical solutions computed via the finite element method ${ }^{136}$ or Monte Carlo methods. ${ }^{126,137,138}$ Computationally efficient Monte Carlo 
platform for structured light applications can be found at http:// mcx.space/. ${ }^{139}$ Solving the inverse problem is not trivial and many different methods have been developed, ${ }^{124,125,128,137}$ but a description of these methods is beyond the scope of this review. Overall, these traditional DOT/FMT methods enable reconstruction of properties (position, absorption/scattering coefficient, and fluorescence yield) in vitro ${ }^{122,132}$ as well as in vivo in small animals ${ }^{140}$ with high sensitivity and relatively high resolution.

Despite the widespread use of DOT/FMT with point sources and detectors, there are many limitations. On the acquisition side, raster-scanning point illumination and detection methods can lead to overly long acquisition times due to the necessity to acquire dense spatial data sets. The use of point sources also limits the power density that can illuminate the sample at each point, to avoid photobleaching in fluorescent samples or damage to tissue for in vivo samples. Moreover, such methodologies are not amenable to scaling to large volumes without loss of volume sampling. On the reconstruction side, since there are many sampled source-detector pairs, the measured data sets become very large as does the sensitivity matrix generated from the forward model used in solving the inverse problem. This results in high computational burden, which limits the postprocessing method despite improvements in hardware and software.

\subsection{Method Development}

Early techniques such as phased array ${ }^{141}$ were proposed to mitigate the above-mentioned issues and boost the sensitivity ${ }^{142}$ and resolution of DOT. ${ }^{143}$ However, these early concepts of structured excitation of tissue are based on photon density wave interference that can be challenging to generate and control. It is nowadays relatively straightforward to generate spatially modulated wide-field illumination and detection methodologies using DMDs to harness the potential of structured light strategies. Hence, researchers have increasingly begun to investigate wide-field detectors and structured illumination to efficiently perform DOT.

As a more general tomographic approach, utilizing structured light of any arbitrary shape can be utilized for tomographic imaging. Some of the main expected benefits of structured light strategies are reduced number of required measurements due to the inherent wide-field nature of structured light and the potential for harnessing compressive sensing methodologies. Indeed, CS methodologies have been developed over the last decade to help reduce the size of digitally large data sets during the acquisition step. Since CS can take advantage of the sparsity of the image sample plane when illuminated with a determined patterned basis, the desired image can be recovered with far fewer measurements. Conveniently, structured light approaches enable one to efficiently implement this by selecting a sparse illumination basis. Therefore, CS allows for data compression during the acquisition step, which is beneficial for acquisition time reduction. ${ }^{144}$ In addition, the samples can be illuminated with a decreased overall power density due to the large illumination areas leading to relaxation of safety and photobleaching issues. Since one can change the shape and intensity at or close to region of interest where the signal is relatively low due to high absorption (e.g., liver in small animal imaging), an increased SNR can be achieved in those areas due to efficient illumination strategy related to the target site.

These implementations come at the cost of slightly increased computational complexity as modeling of spatially complex sources is required in the forward model simulation for generation of the sensitivity matrix [cf. Fig. 5(i)]. However, flexible, accurate, and universal computational Monte Carlo tools are now widely available to generate such sensitivity matrices both in the case of structured illumination as well as detection. ${ }^{139}$

The first investigations of the potential of structured illumination in DOT were performed using simulations. Lukic et al. ${ }^{149}$ proposed the use of structured illumination for tomography in the frequency domain, and they demonstrated in silico that reconstructions performed with structured illumination patterns provided comparable resolution to those generated using pointsource illumination. In addition, they showed that the amount of data acquired using structured light was decreased by a factor of five compared to a previously tested point-source illumination system. Similarly, Joshi et al. ${ }^{150}$ proposed the use of structuredlight tomography in the time-domain in silico but in the reflectance geometry. The researchers compared a scanned line pattern, scanned Gaussian spot patterns, patterns such as a cross, and a series of equally spaced lines that can be generated with diffractive elements, and point sources on simulated phantoms with fluorescence inclusions. They showed that structured illumination patterns outperform point-source illumination in terms of resolution and location accuracy for multiple fluorescent inclusions close to the surface. Each pattern type provided a slightly different reconstruction result, which suggested that the patterns need to be optimized according to the sample of interest. In this regard, Dutta et al. ${ }^{151}$ developed an optimization framework for generating optimal spatial illumination patterns for CW FMT based on an approach that seeks to improve the condition number of the Fisher information matrix. However, this methodology is computationally expensive and requires prior knowledge of sample surface topography and tissue optical properties, which makes it difficult to implement practically in vivo.

These simulation studies paved the way for current implementations of structured-light tomography. Spatial frequencydomain tomography utilizing sinusoidal spatial illumination was first implemented in reflectance geometry by Konecky et $\mathrm{al}^{32}$ by solving the heterogeneous diffusion equation with a linear perturbative approximation in Fourier space. The authors demonstrated the feasibility of fast tomographic reconstruction of absorption contrast due to the analytical expressions based on Green's functions and the Rytov perturbation model. The work was later extended for 3-D reconstructions of fluorescence contrast with the clinical application of surgical guidance of glioma resection (cf. Fig. 5). ${ }^{114}$ For full volume tomography beyond a couple of millimeters, Bélanger et al. ${ }^{146}$ implemented structured-light-based strategies in transmission and compared two initial sets of 36 wide-field patterns, namely checkerboard and low spatial frequency bar patterns that illuminated half of the sample area in each pattern, in a system with structured illumination and wide-field detection. These patterns were tested in centimeters-thick simulated phantoms as well as liquid phantoms containing graphite rods with $100 \%$ absorption. Their results showed that it was possible to reconstruct inclusions with high resolution and accurate locations with a smaller number of measurements compared to the data sets collected with traditional tomography systems, leading to near-real-time acquisition speeds. In addition, it was determined that the set of bar patterns provided better reconstruction of absorption contrast and showed higher robustness to noise compared to the checkerboard pattern set [cf. Fig. 5(ii)]. This 


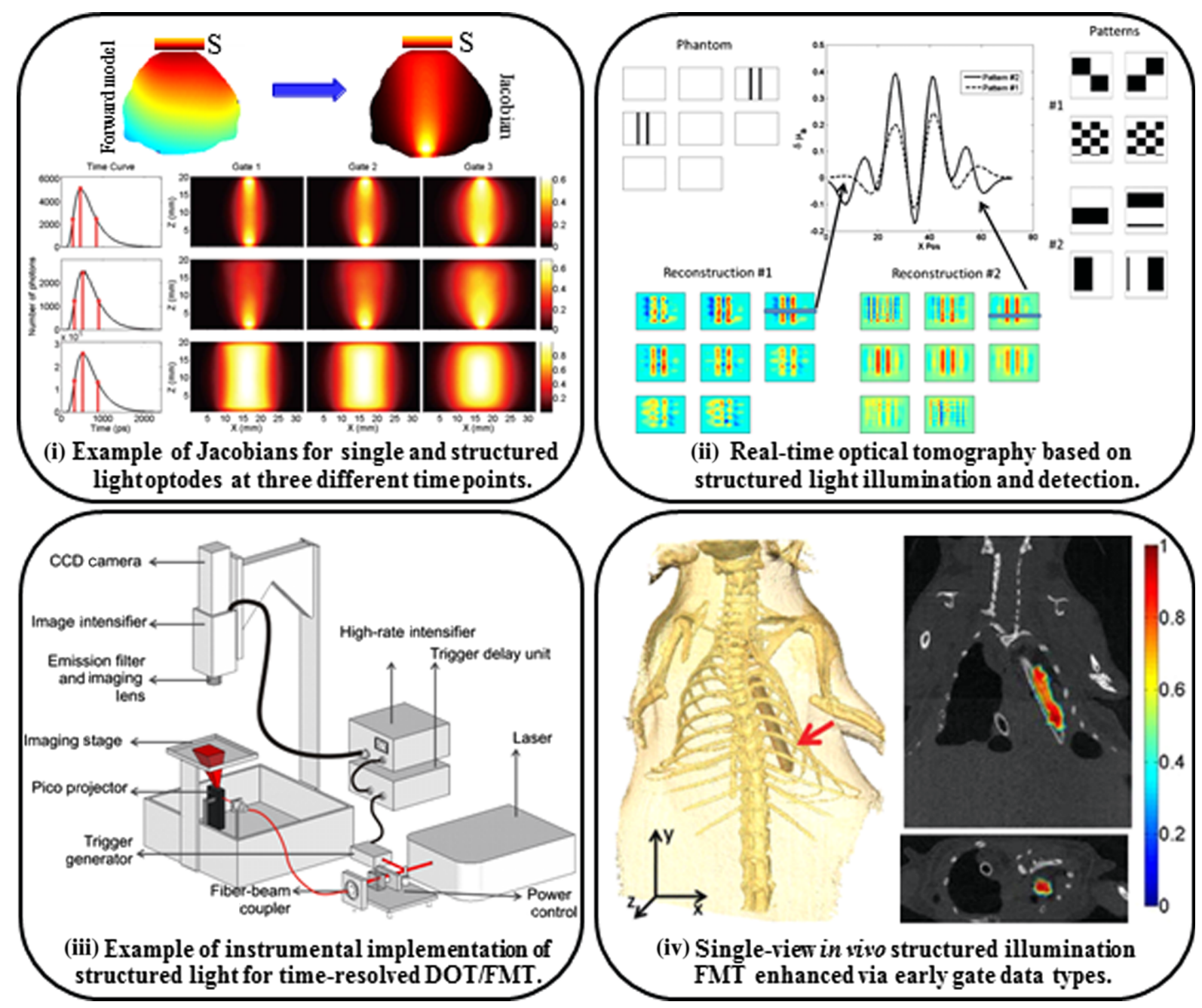

Fig. 5 Light tomographic implementations: (i) simulated detector readings for the central source-pattern and associated Jacobians for the three gates selected: point source-point detector (top row), patterned illumination-point detector (middle row), and patterned illumination-patterned detection (bottom row adapted from Ref. 145). (ii) Simulated phantom reconstructions based on two types of patterns: phantom used for the simulations (top left), two types of patterns for illumination and detection (top right), reconstructions for each pattern type (bottom), cross sections and quantitative values of the differential absorption across a $z$-slice. The volume for the reconstruction is divided in $16 z$-slices, each having $1.25-\mathrm{mm}$ thickness; even $z$-slices are shown (top middle adapted from Ref. 146). (iii) Wide-field fluorescence lifetime imaging system for ex vivo and in vivo imaging. The schematics of the time-domain fluorescence lifetime imaging system based on a gated ICCD detection are shown (from Ref. 147). (iv) 3-D volume from the CT scan showing the position of the tube in the chest cavity (left). Coronal slice of the reconstructed volume at $z=6: 5 \mathrm{~mm}$. (Bottom right) Transverse slice of the volume at $y=21: 5 \mathrm{~mm}$ (top right adapted from Ref. 148).

work was closely followed by the implementation of structured illumination for time-resolved preclinical studies. ${ }^{147}$ The schematic of the system developed by Venugopal et al. is provided in Fig. 5(iii). Using the same bar patterns as Bélanger et al., Chen et al. ${ }^{145}$ demonstrated in vitro that these bar patterns provided accurate reconstruction of absorptive inhomogeneities. Also, leveraging the time-resolved data sets, Venugopal et al. ${ }^{147}$ demonstrated the ability to quantitatively reconstruct both absorption and scattering contrast with minimal cross talk. Lastly, Venugopal et al. ${ }^{152}$ were the first to demonstrate the utility of the technique in imaging fluorescence signals in small animals at fast acquisition speeds. The combination of Monte Carlo-based forward simulations ${ }^{153}$ that enable harnessing of the early photons for improved resolution and late photons for quantification ${ }^{134}$ led to accurate reconstructions even in the case of a single-projection system. ${ }^{154}$ An example of timeresolved enhanced FMT reconstruction is provided in Fig. 5(iv). Ducros et al. ${ }^{128}$ also compared these data types in the reflectance and transmission geometries, and they came to similar conclusions regarding the use of time-resolved data.

The bar patterns used in these pioneering studies were selected based on the work of Bélanger et al. ${ }^{146}$ but also due to the experimental ease of implementation for complex geometries such as in small animal imaging. Still, numerous spatial bases can be considered and implemented such as typically done in the field of compressive sensing. To date, a few studies have investigated the use of well-known bases for structured-light tomography with the goal of improved compression of the 
measurement space, such as measurements obtained with wavelet-based patterns ${ }^{155}$ and piecewise-constant functions. ${ }^{156}$ Moreover, theoretical CS bases typically contain negative components that cannot be directly projected onto the sample. Following well-established imaging protocols in CS-based 2-D imaging, Ducros et al. ${ }^{157,158}$ proposed a virtual source pattern method in which the patterns, which can be negative or have a complex component, are transformed into a projectable pattern with positive intensities using a transformation matrix. This transformation matrix is then applied to the measured data as well as the calculated sensitivity matrix for reconstruction of the inclusion. Methods have also been developed to decrease the overall computation time of tomography, such as the initial projection of sinusoidal fringe patterns to construct a priori surface profiles for generation of the forward model. ${ }^{159-161}$ In addition, structured light strategies are well-suited to benefit from CS-based preconditioning of the illumination and detection fields for improved performance. ${ }^{162}$ Beyond the potential of using optimal illumination patterns based on theoretical considerations, structured light methodologies are also amenable to implementing strategies to improve the SNR of the measured data set. For instance, Zhao et al. ${ }^{163,164}$ demonstrated that by iteratively optimizing the local illumination field, sensitive topographic imaging in preclinical models was achievable. Venugopal and Intes ${ }^{165}$ proposed a similar concept for 3-D imaging and demonstrated that the number of useful data sets could be increased by twofold using this methodology. Such an approach is poised to enable the collection of optimal data sets without requiring any prior knowledge. These developments have laid the foundation for wide dissemination of structured-light tomography. As the technique is adopted by an ever-increasing number of research groups in the world, more applications are benefiting from such improvements.

\subsection{Applications and Further Developments}

\subsubsection{In vivo fluorescence molecular tomography}

FMT has become popular over the last two decades due to improvements in and availability of NIR fluorescent dyes, biomarkers, and reporter genes that can be used in vivo. The three major benefits of FMT for in vivo molecular studies are its high sensitivity that can rival nuclear imaging, its ability to simultaneously image multiple biomarkers via spectral and lifetime encoding, and the unique wealth of information that can be derived from lifetime sensing such as microenvironment parameters or protein-protein interactions. To date, FMT in preclinical settings has been the main application of structured-light tomography.

Venugopal et al. ${ }^{152}$ were first to demonstrate in vivo accurate reconstructions of fluorescent inclusions inserted into a freshly euthanized mouse. The performance of this single-projection structured light approach was cross-validated with nonconcurrent CT scans. Of note is that no a priori information was included in the optical reconstruction. As shown in Fig. 5(iv) in an overlay with coregistered microCT data, the inclusion was reconstructed with high accuracy due to the early gate data type and sparsity-enhancing solvers. ${ }^{154}$ Similarly, Ducros et al. ${ }^{166}$ later demonstrated the use of experimentally adapted virtual source Haar wavelet patterns for FMT of a fluorescent inclusion implanted within a euthanized mouse in a system with a rotating stage. First, the mouse was imaged at multiple rotation angles using a wide-field pattern for generation of adapted patterns as well as the model for forward calculations. These patterns were then used for imaging of the mouse at eight rotation angles to enable reconstruction of the fluorescence inclusion. As shown in Fig. 6(i), the inclusions were accurately reconstructed in terms of the locations and dimensions, but the authors note that there are still artifacts within the reconstruction that can be improved with the use of a priori information. Still in both the cases of Venugopal et al. and Ducros et al., the 3-D data acquisition was performed at fast acquisition speeds and enabled whole-body 3-D imaging.

Demonstration of the ability to image an ex-vivo preclinical model was also demonstrated by Pimpalkhare et al. ${ }^{167}$ The specimen employed was a dog spine model injected with a small dose of indocyanine green (ICG), a common NIR fluorophore. Multiview time-resolved-structured light tomography was performed and accurate distribution of the fluorophore as retrieved in this complex sample was validated by microMRI cross-validation [cf. Fig. 6(iii)]. Interestingly, the authors noted that improvements in resolution were more pronounced when using multiple views compared to leveraging of early gate data sets. However, implementation of multiview systems for preclinical studies is cumbersome and typically done by rotating the sample in a vertical position. This is not a natural physiological posture for animals and leads to challenges when performing nonconcurrent imaging with other common modalities that are designed around a prone position.

There are currently still more efforts to improve efficiency and accuracy of structured light FMT. For instance, noncontact and nonrestrained preclinical imaging leads to the projection of theoretical patterns on complex boundary conditions. SLMs are flexible enough to enable the scaling of patterns in real-time to encompass the boundaries of the animal. Then, to ensure reconstruction accuracy, a priori information about the projected patterns should be included in the forward model via a complex modeling scheme such as a mesh-based method. ${ }^{138,139}$ Current forward solvers such as GPU-enhanced finite element Monte Carlo are particularly adept for such complex modeling. ${ }^{138}$ Moreover, in combination with computationally efficient formulations, ${ }^{168}$ whole Jacobians, even in the timeresolved cases, can be computed in a matter of minutes on a personal computer. Combined with mesh optimization techniques, ${ }^{169}$ they offer the unique attributes of accuracy for all kinds of diffuse regimes, fast computational times, and improved resolution via mesh refinement.

\subsubsection{Lifetime-based tomography}

Beyond establishing the potential of structured-light tomography for retrieving the biodistribution of fluorescent markers, Venugopal et al. ${ }^{148}$ demonstrated the utility of the methodology to image lifetime-based contrast. Fluorescence lifetime can be defined as the intrinsic property of a fluorophore of reaching an excited state that will lead to the emission of photons, to then return to its initial ground state. ${ }^{170}$ Since it is an intrinsic property, it is independent of concentration, tissue depth, or photobleaching, being only affected by extrinsic factors such as temperature or quenching. ${ }^{170}$ More precisely, the authors demonstrated the ability of time-resolved structured-light tomography to quantify Förster resonance energy transfer (FRET) occurrence via sensing of the donor lifetime.

In brief, FRET is a phenomenon that occurs when two molecules that have high spectral overlap, denoted the donor and acceptor, are within 2 to $10 \mathrm{~nm}$ apart. At this distance, 


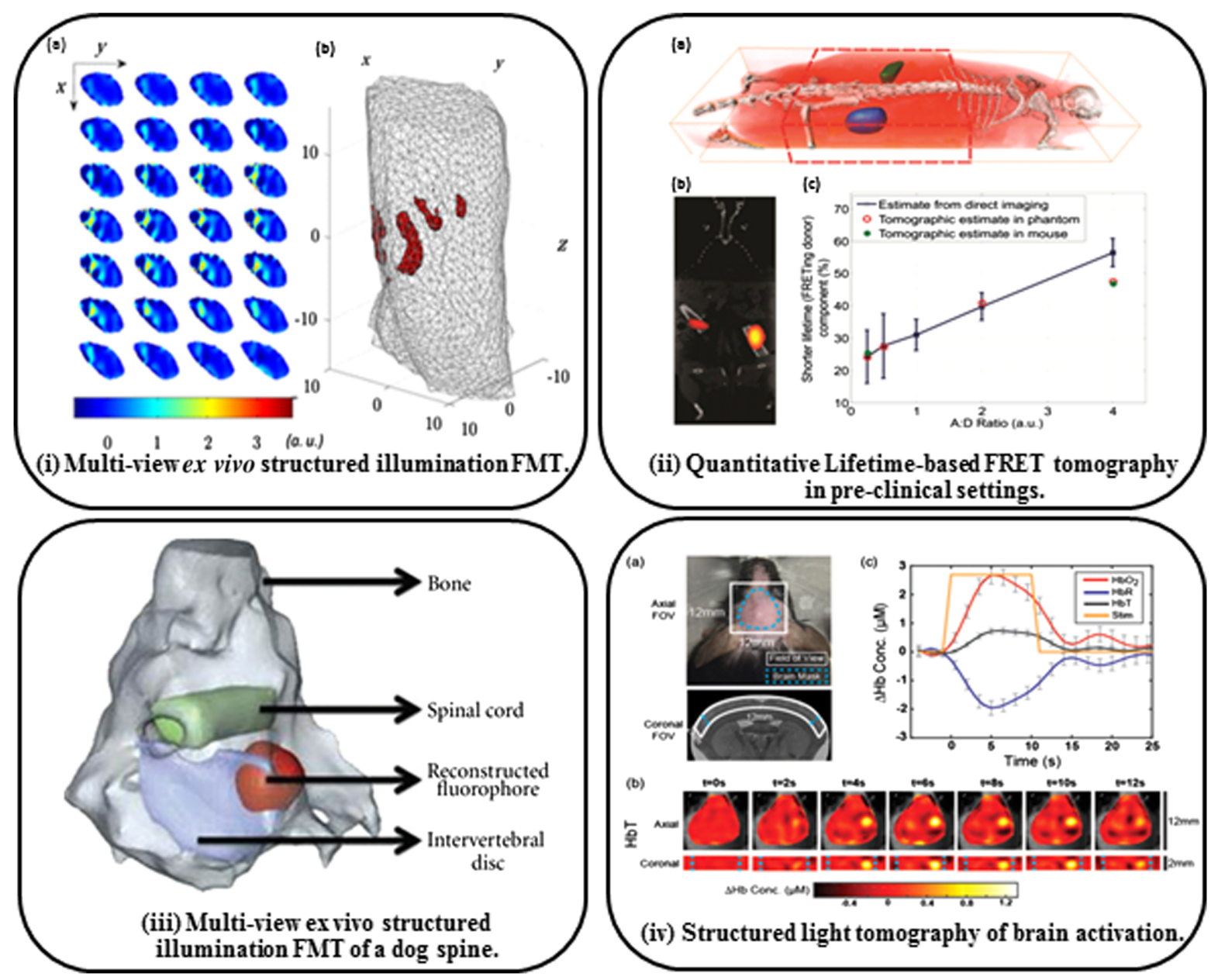

Fig. 6 Applications of tomographic reconstruction using structured illumination: (i) of fluorescent tubes implanted within a freshly euthanized mouse. The data shown were taken by moving low spatial frequency quantized bar pattern illumination and wide-field detection. The data shown were collected with virtual source Haar wavelet patterns, (a) the raw fluorescence data for multiple views and (b) the reconstructed inclusions within the $3-D$ mesh. $^{166}$ (ii) Tomographic quantification of FRET. Reconstruction of the fluorescence quantum yield is shown in (a) overlay onto a coregistered microCT model in 3-D and (b) in a cross-sectional slice. (c) The quantification of FRET through use of the FRETing donor fraction of the two FRET inclusions is similar to the values extracted from tomographic imaging of the mouse, a separate phantom with four FRET inclusions, and direct planar imaging. ${ }^{148}$ (iii) Reconstructions of ICG distribution in an ex vivo dog spine using multiview time-resolved structured light illumination and mesh-based Monte Carlo with nonconcurrent MRI. ${ }^{167}$ (iv) Structured light tomography of brain activation in a live mouse. (a) The imaging area is shown in axial and coronal slices. The paw of the mouse was stimulated at the beginning of the imaging session, and (b) the distribution of total hemoglobin $\mathrm{HbT}$ within the brain was seen to change according to activation of various areas of the brain. (c) The time-course response in terms of total hemoglobin, oxygenated hemoglobin $\left(\mathrm{HbO}_{2}\right)$, and deoxygenated hemoglobin $(\mathrm{HbR}) .{ }^{171}$

the donor transfers energy to the acceptor, which causes the intensity and lifetime of the donor to decrease while those of the acceptor increase. By measuring the changes in intensity or lifetime of the donor, FRET can be used as a nanoscale proximity assay in vivo. Lifetime-based FRET has already been established as a useful tool for monitoring ligand-receptor engagement in vivo in wide-field planar imaging of cancerous tissue. ${ }^{172-174}$ Tomography of FRET was validated by Venugopal et al. ${ }^{148,175}$ using an NIR FRET pair, AF700-AF750. For the in vivo study, the moving low spatial frequency bar patterns optimized with the adaptive optimization method ${ }^{165}$ were utilized on a freshly euthanized mouse with two inclusions of different FRET ratios. As shown in Fig. 6(ii), the locations and fluorescence yields of the two inclusions were accurately reconstructed. In addition, it is shown that the tomographic reconstruction of the donor population that interacts with the acceptor (denoted the FRETing donor and characterized by a quenched lifetime) is consistent with the two inclusions from tomographic reconstruction of FRET in a phantom experiment as well as from direct nontomographic imaging. The quantification of the FRET donor via tomographic imaging was reported to be within $5 \%$ of the value quantified on the same system but without any surrounding scattering tissue.

\subsubsection{Functional imaging}

Structured-light tomography is also well-suited to image endogenous markers. The improvement in acquisition speed positions 
the technology favorably for any applications targeting hemodynamics. This is a nascent application with great promise. Recently, Reisman et al., ${ }^{171}$ following the pioneering developments highlighted above, implemented structured-light tomography for functional brain imaging of the intact mouse brain (through the scalp and skull). The researchers collected the data by projecting sinusoidal patterns of different spatial frequencies, phases, and orientations. The sensitivity matrix was generated using a finite element mesh and the diffusion equation, and the local absorptive perturbations associated with modulation of the hemodynamics were reconstructed. As shown in Fig. 6(iv), changes in blood flow were realized upon stimulation of a limb, which was characteristic of activation of the specified brain region. This method is especially powerful since it allows for monitoring of different hemodynamic properties noninvasively in a live mouse. Imaging of brain activation has typically been performed using NIR spectroscopy, but incorporating structured-light tomography shows improved recovery of focal brain functional activation. Such implementations may find numerous other applications in functional imaging such as optical mammography ${ }^{176}$ or monitoring of perivascular diseases.

\section{Single-Pixel Imaging}

\subsection{Introduction}

Another rising application of structured light strategies in diffuse optics is the implementation of single-pixel camera methodologies. The combination of SLMs with compressed-sensing approaches enables the development of imaging systems based on one detector that nevertheless can provide 2-D imaging capabilities without any moving parts. Even though CCD and CMOS light sensors have improved throughout the years, their application for spectral bands beyond the visible regime is still limited since they mostly operate in the visible range and producing them for the NIR and infrared range is complex and expensive. ${ }^{177}$ Conversely, single-pixel systems can leverage detectors sensitive to these spectral bands and provide 2-D imaging capabilities at a reduced cost. The standard setup of a single-pixel system is composed of a DMD, which is used to project patterns onto the sample plane, and a single-pixel detector that collects the sample emissions after illumination. This classical setup has been often modified into more complex systems with enhanced detectors or illumination schemes. These systems can also adopt different scanning methodologies that can yield better intensity reconstructions or improved acquisition times. ${ }^{177}$

As described by Welsh et al., ${ }^{178}$ ghost imaging, the precursor of single-pixel imaging, initially consisted of two detectors with low and high resolutions, respectively. The first one collected the light scattered from the sample and the second one detected the unscattered illumination scheme. The incident light could be generated by pseudothermal light sources ${ }^{179}$ that produced speckle-like illumination. A beam splitter would duplicate the illumination arrangement for it to be sampled by the highresolution detector. The data collected from both detectors could later be used to retrieve the sample's image. The use of this detector-beam splitter complex was later unnecessary once SLMs were introduced. An SLM would computationally produce structured illumination where parameters such as phase and intensity were regulated. Therefore, the use of known illumination fields removed the need of a high-resolution detector. Hence, the setup was simplified to one detector and an SLM for illumination, which is the basic scheme of a single-pixel detection system. Combining the single-pixel detection scheme with compressive sensing, ${ }^{177}$ which takes advantage of the sparseness of the acquired data, reduces the amount of required data to reconstruct the sample's image.

The main advantages of single-pixel implementation are twofold. First, compared to other imaging schemes that depend on expensive pixelated detectors that respond only to visible wavelengths of light, single-pixel imaging uses a single detector which can, when selected judiciously, sense desired wavelengths outside the visible range. ${ }^{180}$ Numerous applications in the biomedical field can benefit from cheaper and better sensitivity in these spectral bands, ${ }^{7,181}$ such as infrared imaging, ${ }^{182}$ hyperspectral imaging, ${ }^{183}$ or $3-\mathrm{D}$ imaging. ${ }^{184,185}$ Second, single-pixel detectors can work better under poor light conditions. ${ }^{181}$ This is mainly associated with more sensitive detectors but also due to the spatial integration of the optical signals collected at each illumination pattern. It is also of note that single-pixel systems can be extended to the timedomain for efficient wide-field lifetime imaging applications. The basic setup can be enhanced by adding a time-correlated single-photon-counting unit (TCSPC), resulting in a time-correlated single-pixel system. ${ }^{183}$ Time-correlated systems can be later analyzed to investigate properties such as fluorescence lifetime ${ }^{186}$ or FRET. ${ }^{187}$ Unfortunately, in the classical singlepixel detection configuration, a high number of patterns are needed to yield a good image reconstruction, which also correlates to higher exposure times for the sample. Moreover, the quality of the reconstructed images is lower than the one obtained using a common pixelated detector. These disadvantages have been overcome through the years by fusing the technique with theories such as compressive sensing.

\subsection{Methods}

A single-pixel system typically follows the design proposed by Duarte et al. ${ }^{177}$ with slight modifications based on the application at hand. Overall, a single-pixel camera is composed of a single-pixel detector, an SLM for pattern illumination, and relay optics as shown in Fig. 7(i) by Rousset et al. ${ }^{181}$ The SLM typically is a DMD, which gives the advantage of producing both binary and grayscale structured illumination. The angle of the DMD micromirrors can be digitally manipulated to filter a determined amount of light for that specific area. All the mirrors in conjunction can then produce grayscale patterns with up to 10-bit resolution. ${ }^{181}$ The characteristics of the basic components are dependent on the application of the system. The detectors can vary from "bucket" detectors ${ }^{189}$ that have no spatial resolution and directly detect all the scattered photons, e.g., single photodiodes and photomultiplier tubes, to multichannel detectors $^{187}$ that detect dispersed light in multiple wavelength channels. The relay optical elements vary depending on the type of detector and illumination scheme, for example, they can adopt lensless configurations ${ }^{190,191}$ or use elements such as physical masks. ${ }^{192}$ Optical setups can also vary from single-pixel microscopy systems ${ }^{193,194}$ to macroscopic approaches. ${ }^{188,195}$

\subsubsection{Scanning methodologies}

Beyond the consideration of the best optical elements and detectors for the application at hand, one must also select a proper scanning methodology. Indeed, as mentioned by Duarte et al. ${ }^{177}$ a single-pixel camera can operate under different 


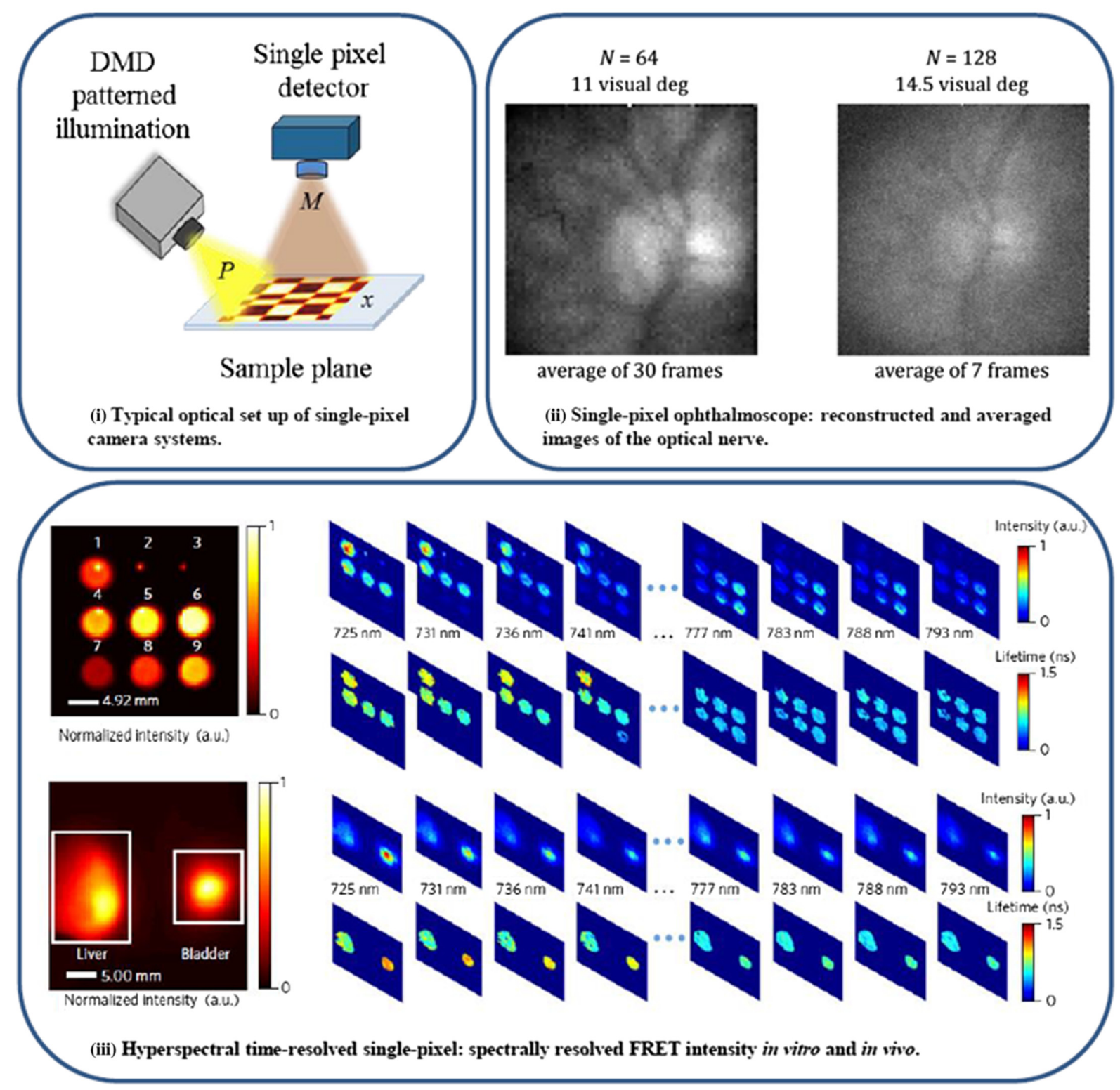

Fig. 7 (i) Simplified optical setup of the single-pixel camera using a DMD. The image is noted $x, P$ is a DMD pattern, and $M$ is the corresponding measurement. ${ }^{181}$ (ii) Living human eye imaging using a singlepixel camera ophthalmoscope. ${ }^{188}$ (iii) Spectrally resolved FRET intensity imaging in vitro and in vivo. ${ }^{1{ }^{17}}$

acquisition methodologies such as the basic principle of acquiring $N$ measurements to reconstruct an $N$-resolution image or under multiplexing strategies such as compressive sensing. These scanning methodologies must take into consideration the signal-to-noise ratio during the acquisition process, which can be diminished by Poisson noise or the general instrument response. If an $\mathrm{N}$-pixel image is to be reconstructed through single-pixel measurements, it can be typically done by using a raster scan, a pixel array, or a basis scan. In the latter case, basis scanning is constructed using a single sensor that will detect $N$ measurements acquired one after the other with patterned illumination, where the patterned light will target diverse arrangements of the $N$ pixels on the image plane. ${ }^{177}$ Multiple types of illumination basis can be employed, the most commonly used include Hadamard, speckle, Fourier, and orthogonal, or biorthogonal wavelets such as Haar, LeGall, and Daubechies. Furthermore, basis scanning can take advantage of the image plane sparseness to only require part of the full basis patterns to reconstruct the image plane through compressive sensing algorithms. ${ }^{177,196,197}$ Basis scanning performs better than raster scanning by reconstructing similar intensity images at much lower capture times. ${ }^{177}$ Therefore, compressive sensing reduces the number of patterns per total acquisition, consequently reducing the exposure time. This is key in cases where the sample is sensitive to photodamage within the expected complete acquisition time.

\subsubsection{Image recovery approaches}

For single-pixel camera systems, the 2-D image sought is not obtained via direct imaging but via an inverse problem. Indeed, the data acquired during basis scanning with a single-pixel camera is the inner product of the illumination patterns and the sample, therefore retrieving the sample's intensity profile through processing algorithms is necessary. This inverse problem is rather simple and can be expressed as 
$M=\Delta t P x$,

where $M$ is the single-pixel measurement over a $\Delta t$ acquisition period, $P$ denotes the set of illumination patterns used for acquisition, and $x$ represents the image of the sample plane. ${ }^{181}$ Compressive sensing assumes that the sample can be sparsely represented by a basis $\alpha$. Therefore, $x=\alpha s$, where $s$ represents the sparse image plane. Note that the illumination basis has to satisfy the "restricted isometry property."198 Solving a minimization-optimization problem for that particular transformdomain can then retrieve the sample's image. ${ }^{181}$

\subsubsection{Pattern selection}

One area of focus for the field of single-pixel imaging is the selection of optimal basis for fast and accurate 2-D image reconstructions. Common bases include Hadamard, speckle, Fourier, and wavelet-based patterns. Streeter et al. ${ }^{199}$ explained that Hadamard multiplexing is known to improve the SNR in the acquired data by decreasing additive noise. Two main types of Hadamard matrices are commonly used, the H-matrix which is composed of ones (1's) and minus ones ( -1 's) and the S-matrix which is formed by zeros (0's) and ones (1's). Since the S-matrix is easier to implement in SLMs, it is more commonly employed for structured illumination than the H-matrix, even though the latter should provide an even better SNR boost. Although the additive noise is decreased, Hadamard patterns are affected by Poisson noise generated by photons, which can decrease the SNR of the measurements. ${ }^{199-201}$

As described by Guo et al., ${ }^{191}$ speckle patterns can also be implemented in single-pixel systems, where the resolution of the reconstructed image will be dependent on the size of the speckle features. Speckle patterns are nonuniform illumination, which have been previously used in microscopy systems to improve the image resolution beyond the diffraction limit. ${ }^{191,202}$

According to Zhang et al., ${ }^{180}$ Fourier patterns can also be employed for the image acquisition process. When compared to Hadamard patterns, Fourier patterns are more efficient, while Hadamard patterns are more resistant to noise. The efficiency of Fourier patterns comes from their ability to concentrate the energy of the image plane. In addition, using multistep Fourier illumination in certain cases can help reduce the number of measurements and therefore the acquisition time.

Rousset et al. ${ }^{181}$ assert that since the compressive sensing reconstruction process based on minimization is computationally expensive, approaches that could yield better reconstruction times are necessary, especially for applications where a fast acquisition time is necessary. Adaptive basis scanning by wavelet prediction has been proposed to allow a more straightforward image recovery than fundamental compressive sensing. This approach involves the use of a wavelet basis such as Haar or LeGall for illumination and is based on predicting the most significant set of patterns to be used for a particular image plane. For the prediction process, a set of initial single-pixel measurements with low-resolution patterns needs to be acquired. This data set can later be used for predicting the significant wavelet coefficients of the image. These coefficients are indexed to specific patterns that will be further used for the main acquisition process. The use of wavelet patterns is justified by the fact that most images can be sparsely characterized in this basis and by the accessibility to inverse wavelet algorithms for image reconstruction. ${ }^{181,203,204}$
Another approach that has been proposed to reduce computational time for the image reconstruction process is adaptive compressive sensing imaging. Adaptive approaches have been pursued since the use of basis will often involve measuring background pixels that are not within the sample's region of interest. If the acquisition patterns can be selected to target the interest area from the sample, the acquisition times may be lowered and the reconstruction process enhanced.

Soldevila et al. ${ }^{189}$ indicated that methods that are based on $a$ priori information can be disadvantageous in cases where the imaged sample can unpredictably change. Even though these techniques are beneficial because they define regions of interest on the image plane, it would be better to have a method where no a priori information is needed. Adaptive compressive imaging is based on recovering the image under a compressive sensing approach that defines regions of interest during the acquisition process but without the need for a priori information. The method involves using sets of masks that will adaptively change depending on the regions of interest on the image plane. The masks can iterate in size depending on the desired resolution. Like other acquisition techniques, it uses inverse wavelet transforms for the image reconstruction process. ${ }^{189}$ The small acquired matrices can then be processed without the need of high computational demand. The image plane is first sampled and delimited by low-resolution masks. Then, a one-level wavelet transform and an edge detection algorithm discard the borderless regions and determine the regions of interest. The high-resolution masks are only applied to these areas. This process can be repeated until the high-resolution masks are only applied to the areas with finer details. Further improvements are still desired on the recognition algorithms to increase the efficiency of the process.

\subsection{Applications}

Single-pixel imaging can be used to monitor fluorescence lifetime. ${ }^{205}$ In the medical imaging field, this new modality has found applications in microscopy ${ }^{206}$ as well as in macroscopic systems. ${ }^{207}$ Pian et al. ${ }^{187}$ reported a single-pixel system that combines hyperspectral detection in the time-domain. A multichannel detector is coupled to a TCSPC unit to perform macroscopic fluorescence lifetime imaging at different detection wavelengths. The structured illumination is produced by DMDs arranged in a transmission or reflection configuration. The system uses the compressive sensing multiplexing scanning methodology and utilizes Hadamard basis as the illumination pattern set. The system has been employed to quantify and image fluorescence lifetime both in vitro for tissue-simulating phantoms and in vivo for mice. Macroscopic fluorescence lifetime imaging is highly sensible and serves to "unmix" the fluorescence lifetime values of different biomarkers. In addition, the described system ${ }^{187}$ has been used to measure FRET, which describes the interactions of the sample at the molecular level by quantifying the ratio of donor molecules to acceptor molecules and estimating the distance between them. ${ }^{208}$ Figure 7(iii) highlights results obtained from FRET-fluorescence lifetime imaging. Pian et al. ${ }^{195}$ also reported a single-pixel imaging system that can be used for time-resolved hyperspectral tomographic imaging when combining DMD detection and illumination structures in transmission geometry. It has been used to map the concentration of targeted fluorophores.

Finally, Lochocki et al. ${ }^{188}$ proposed that single-pixel imaging systems can be an alternative to the ophthalmoscopes that are 
necessary to determine a variety of eye illnesses. Even though ophthalmoscopes have been improved throughout the years, they are not fully useful in cases where the ocular structures are opaque. As proposed, a single-pixel camera approach was implemented to image the retina in real-time. The technique was validated in human and artificial eyes to image the fundus of the eye at $15 \mathrm{deg}$ of visual angle. The results obtained from artificial eyes, shown in Fig. 7(ii), highlight the potential of the technique for this application, yet further improvement is needed to correct for eye movements on human subjects.

\section{Conclusions}

The benefits of utilizing spatially structured light projection and collection are manifold, stemming from the ability to create nearly any desired set of spatial input or output configurations in a fast, repeatable manner. This ability helped push the development of spatial frequency-domain photon propagation theory from imaging 22 to tomographic reconstruction ${ }^{32}$ with contemporaneous development of single-pixel imaging. ${ }^{177}$ Since 2010, diffuse optics has been burgeoning with structured-light techniques taking a lead role in its progress.

Quantitative, noncontact imaging techniques have since been developed for real-time, wide-field acquisition ${ }^{38-40}$ and have been pushed toward endoscopic implementation. ${ }^{80,81}$ Models have also been moving toward new speeds and higher accuracy $^{49}$ while becoming robust via subdiffuse sensing. . $^{4,47}$ Many developments in modeling, processing, and instrumentation have helped push efforts toward clinical efficacy, combining SFDI with modalities such as laser speckle, ${ }^{89,90}$ laser Doppler, ${ }^{11}$ and polarimetric imaging ${ }^{82}$ to accurately predict burn wound severity within $1 \mathrm{~h}$ of infliction. Brain imaging with SFDI techniques have demonstrated the potential for identifying Alzheimer's disease, ${ }^{84,112}$ its correlated neuronal death, ${ }^{113}$ and for fluorescence-guided tumor resection. ${ }^{114,115}$ Oxygenation sensing with SFDI has demonstrated potential in clinical utility for imaging breast cancer, ${ }^{74}$ skin cancer, ${ }^{56,116,209}$ skin flap viability, ${ }^{107,210}$ and has just gained FDA approval for identifying lower limb vascular issues. ${ }^{75}$

DOT has seen new growth due to the flexibility and precision of DMDs. They enable rapid pattern generation for illuminationdetection pairs to be sampled fast enough for real-time tomographic sensing ${ }^{146}$ and are sensitive enough for in vivo reconstructions with high fidelity. ${ }^{152}$ Rapid acquisition has been coupled with GPU processing ${ }^{138}$ and computationally efficient formulations for reconstructions that take mere minutes on a personal computer. ${ }^{168,169}$ The use of FRET with structuredlight tomography has pushed this limit further and has been demonstrated as a nanoscale proximity assay in small animals. ${ }^{148,165}$ Endogenous sensing continues to improve with structured light, as real-time tomographic maps of cerebral hemodynamics in a mouse (through the scalp and skull) ${ }^{171}$ are possible and mammogram reconstructions improve. ${ }^{176}$

Single-pixel imaging with a point detector allows for low light level detection and has much greater flexibility than 2-D detection in the chosen optical frequency and bandwidth. Models continue to optimize the selection of illumination patterns for increased image resolution and speed, ${ }^{181,189}$ while also blending into instrumentation to enable lensless imaging. ${ }^{190,191}$ Expanding upon and combining these advantages, single-pixel imaging is now capable of hyperspectral, real-time sensing for cancer detection. ${ }^{207}$ In addition, researchers have combined those attributes with fluorescence lifetime imaging and achieve
3-D reconstruction to reach the state-of-the-art in diffuse optical imaging. ${ }^{187}$

The goal of these diffuse optical technologies is to provide quantitative measurements to clinical and preclinical studies. Of these, 2-D imaging techniques such as those based on SFDI have simpler instrumentation and a much simpler model than that of 3-D methods, allowing it to develop more quickly into physiological studies. However, the lack of depth resolution can be a confounding factor for certain layered media, ${ }^{76,115,117}$ and so the improvement and utilization of 3-D imagers could greatly enhance our understanding of optical contrast in research and clinical settings. On the other hand, commercially available instruments continually improve, making the 2-D imagers more robust, affordable, and capable every year; impressive studies have been done using even office supply equipment. ${ }^{70,72}$ As technology simplifies, it is then available for integration with clinical instrumentation such as endoscopes ${ }^{78,81,116,211}$ and begins to provide quantitative imaging in vivo, in real-time where was once impossible.

\section{Disclosures}

The authors have no financial conflicts of interest to disclose.

\section{Acknowledgments}

This work was supported by the NIH R01 EB019443 and NIH R01 BRG CA207725 (X.I., S.-J.C., M.O.), the National Research Council (NRC) Fellowship (J.P.A.), NIH R21EB01914701 and OIRAIN (U.S.), and the European Research Council (ERC) under the European Union's Horizon 2020 research and innovation program under Grant Agreement No. 715737 (QuantSURG) (S.G.). Certain commercial materials and equipment are identified in order to adequately specify the experimental procedure or reference relevant commercial development. Such identification does not imply recommendation by the National Institute of Standards and Technology.

\section{References}

1. R. Bright, "Reports of medical cases selected with a view of illustrating the symptoms and cure of diseases by reference to morbid anatomy," in Diseases of the Brain and Nervous System, Part II, Vol. II, p. 538, London, Longman, Rees, Orme, Brown \& Green (1831).

2. M. Cutler, "Transillumination as an aid in the diagnosis of breast lesions," Surg. Gynecol. Obstet. 48, 721 (1929).

3. G. A. Millikan, "Photoelectric methods of measuring the velocity of rapid reactions: III-A portable micro-apparatus applicable to an extended range of reactions," Proc. R. Soc. A 155, 277-292 (1936).

4. G. A. Millikan, "Muscle hemoglobin," Physiol. Rev. 19, 503-523 (1939).

5. C. Gros, Y. Quenneville, and Y. Hummel, "Diaphanologie mammaire," J. Radiol. Electrol. Med. Nucl. 53(7), 297-302 (1972).

6. E. Carlsen, Diagnostic Imaging, p. 28, Spectrascan, Inc., South Windsor, Connecticut (1982).

7. S. L. Jacques, "Optical properties of biological tissues: a review; Corrigendum,” Phys. Med. Biol. 58(14), 5007-5008, R37-R61 (2013).

8. B. Monsees, J. M. Destouet, and D. Gersell, "Light scan evaluation of nonpalpable breast lesions," Radiology 163(2), 467-470 (1987).

9. B. Monsees, J. M. Destouet, and W. G. Totty, "Light scanning versus mammography in breast cancer detection," Radiology 163(2), 463465 (1987).

10. A. Ishimaru, Wave Propagation and Scattering in Random Media, Academic Press, San Diego (1978).

11. M. S. Patterson, B. Chance, and B. C. Wilson, "Time resolved reflectance and transmittance for the noninvasive measurement of tissue optical properties," Appl. Opt. 28(12), 2331-2336 (1989). 
12. F. F. Jöbsis, "Noninvasive, infrared monitoring of cerebral and myocardial oxygen sufficiency and circulatory parameters," Science 198(4323), 1264-1267 (1977).

13. D. A. Boas et al., "The accuracy of near-infrared spectroscopy and imaging during focal changes in cerebral hemodynamics," Neuroimage 13(1), 76-90 (2001).

14. S. R. Arridge, "Optical tomography in medical imaging," Inverse Prob. 15(99), R41-R93 (1999).

15. D. A. Boas et al., "Simultaneous imaging and optode calibration with diffuse optical tomography," Opt. Express 5(23), 1143-1158 (1999).

16. X. Cheng and D. Boas, "Diffuse optical reflection tomography using continuous wave illumination," Opt. Express 3(3), 118-123 (1998).

17. A. Kienle et al., "Noninvasive determination of the optical properties of two-layered turbid media," Appl. Opt. 37(7), 779-791 (1998).

18. V. A. Markel and J. C. Schotland, "Inverse problem in optical diffusion tomography I Fourier-Laplace inversion formulas," J. Opt. Soc. Am. A 18(6), 1336-1347 (2001).

19. M. A. A. Neil et al., "Method of obtaining optical sectioning by using structured light in a conventional microscope," Opt. Lett. 22(24), 1905-1907 (1997).

20. N. Dögnitz and G. Wagnières, "Determination of tissue optical properties by steady-state spatial frequency-domain reflectometry," Lasers Med. Sci. 13(1), 55-65 (1998).

21. D. J. Cuccia et al., "Modulated imaging: quantitative analysis and tomography of turbid media in the spatial-frequency domain," Opt. Lett. 30(11), 1354-1356 (2005).

22. D. J. Cuccia et al., "Quantitation and mapping of tissue optical properties using modulated imaging," J. Biomed. Opt. 14(2), 024012 (2009).

23. L. O. Svaasand et al., "Tissue parameters determining the visual appearance of normal skin and port-wine stains," Lasers Med. Sci. 10(1), 55-65 (1995).

24. L. O. Svaasand et al., "Reflectance measurements of layered media with diffuse photon-density waves: a potential tool for evaluating deep burns and subcutaneous lesions," Phys. Med. Biol. 44(3), 801-813 (1999).

25. A. Liemert and A. Kienle, "Spatially modulated light source obliquely incident on a semi-infinite scattering medium," Opt. Lett. 37(19), 4158-4160 (2012).

26. S. C. Kanick et al., "Sub-diffusive scattering parameter maps recovered using wide-field high-frequency structured light imaging," Biomed. Opt. Express 5(10), 3376-3390 (2014).

27. J. Mertz, Introduction to Optical Microscopy, Roberts and Company, Greenwood Village, Colorado (2010).

28. T. A. Erickson et al., "Lookup-table method for imaging optical properties with structured illumination beyond the diffusion theory regime," J. Biomed. Opt. 15(3), 036013 (2010).

29. A. R. Gardner and V. Venugopalan, "Accurate and efficient Monte Carlo solutions to the radiative transport equation in the spatial frequency domain," Opt. Lett. 36(12), 2269-2271 (2011).

30. J. Angelo et al., "Ultrafast optical property map generation using lookup tables," J. Biomed. Opt. 21(11), 110501 (2016).

31. J. Wiest et al., "Polarization influence on reflectance measurements in the spatial frequency domain," Phys. Med. Biol. 60(15), 5717-5732 (2015).

32. S. D. Konecky et al., "Quantitative optical tomography of sub-surface heterogeneities using spatially modulated structured light," Opt. Express 17(17), 14780-14790 (2009).

33. A. Mazhar et al., "Structured illumination enhances resolution and contrast in thick tissue fluorescence imaging," J. Biomed. Opt. 15, 010506 (2010).

34. R. B. Saager, D. J. Cuccia, and A. J. Durkin, "Determination of optical properties of turbid media spanning visible and near-infrared regimes via spatially modulated quantitative spectroscopy," J. Biomed. Opt. 15(1), 017012 (2010).

35. A. Mazhar et al., "Wavelength optimization for rapid chromophore mapping using spatial frequency domain imaging," J. Biomed. Opt. 15(6), 061716 (2010).

36. S. Gioux et al., "Three-dimensional surface profile intensity correction for spatially-modulated imaging," J. Biomed. Opt. 14(3), 034045 (2009).

37. J. Q. Nguyen et al., "Effects of motion on optical properties in the spatial frequency domain,” J. Biomed. Opt. 16(12), 126009 (2011).
38. J. Vervandier and S. Gioux, "Single snapshot imaging of optical properties," Biomed. Opt. Express 4, 2938-2944 (2013).

39. M. van de Giessen, J. P. Angelo, and S. Gioux, "Real-time, profilecorrected single snapshot imaging of optical properties," Biomed. Opt. Express 6(10), 4051-4062 (2015).

40. K. P. Nadeau, A. J. Durkin, and B. J. Tromberg, "Advanced demodulation technique for the extraction of tissue optical properties and structural orientation contrast in the spatial frequency domain," J. Biomed. Opt. 19(5), 056013 (2014).

41. N. Bodenschatz et al., "Model-based analysis on the influence of spatial frequency selection in spatial frequency domain imaging," Appl. Opt. 54(22), 6725 (2015).

42. F. Bevilacqua and C. Depeursinge, "Monte Carlo study of diffuse reflectance at source-detector separations close to one transport mean free path," J. Opt. Soc. Am. A 16(12), 2935 (1999).

43. S. Chamot et al., "Physical interpretation of the phase function related parameter $\gamma$ studied with a fractal distribution of spherical scatterers," Opt. Express 18(23), 23664 (2010).

44. N. Bodenschatz et al., "Detecting structural information of scatterers using spatial frequency domain imaging," J. Biomed. Opt. 20(11), 116006 (2015).

45. D. M. McClatchy et al., "Wide-field quantitative imaging of tissue microstructure using sub-diffuse spatial frequency domain imaging," Optica 3(6), 613-621 (2016).

46. D. M. McClatchy et al., "Monochromatic subdiffusive spatial frequency domain imaging provides in-situ sensitivity to intratumoral morphological heterogeneity in a murine model," J. Biophotonics 10(2), 211-216 (2017).

47. N. Bodenschatz et al., "Quantifying phase function influence in subdiffusively backscattered light," J. Biomed. Opt. 21(3), 035002 (2016).

48. S. D. Konecky et al., "Imaging scattering orientation with spatial frequency domain imaging," J. Biomed. Opt. 16(12), 126001 (2011).

49. A. Liemert and A. Kienle, "Exact and efficient solution of the radiative transport equation for the semi-infinite medium," Sci. Rep. 3(1), 2018 (2013).

50. U. A. Gamm et al., "Quantification of the reduced scattering coefficient and phase-function-dependent parameter $\gamma$ of turbid media using multidiameter single fiber reflectance spectroscopy: experimental validation," Opt. Lett. 37(11), 1838-1840 (2012).

51. S. C. Kanick et al., "Method to quantitatively estimate wavelengthdependent scattering properties from multidiameter single fiber reflectance spectra measured in a turbid medium," Opt. Lett. 36(15), 2997-2999 (2011).

52. K. W. Calabro and I. J. Bigio, "Influence of the phase function in generalized diffuse reflectance models: review of current formalisms and novel observations," J. Biomed. Opt. 19(7), 075005 (2014).

53. D. Yudovsky and A. J. Durkin, "Hybrid diffusion and two-flux approximation for multilayered tissue light propagation modeling," Appl. Opt. 50(21), 4237-4245 (2011).

54. D. Yudovsky and A. J. Durkin, "Spatial frequency domain spectroscopy of two layer media," J. Biomed. Opt. 16(10), 107005 (2011).

55. R. B. Saager et al., "Method for depth-resolved quantitation of optical properties in layered media using spatially modulated quantitative spectroscopy," J. Biomed. Opt. 16(7), 077002 (2011).

56. R. B. Saager et al., "In vivo measurements of cutaneous melanin across spatial scales: using multiphoton microscopy and spatial frequency domain spectroscopy," J. Biomed. Opt. 20(6), 066005 (2015).

57. R. B. Saager et al., "In vivo isolation of the effects of melanin from underlying hemodynamics across skin types using spatial frequency domain spectroscopy," J. Biomed. Opt. 21(5), 057001 (2016).

58. N. Bodenschatz et al., "Surface layering properties of intralipid phantoms," Phys. Med. Biol. 60(3), 1171-1183 (2015).

59. N. Bodenschatz et al., "Sources of errors in spatial frequency domain imaging of scattering media," J. Biomed. Opt. 19(7), 071405 (2014).

60. Y. Zhao et al., "Angle correction for small animal tumor imaging with spatial frequency domain imaging (SFDI)," Biomed. Opt. Express 7(6), 2373-2384 (2016).

61. K. P. Nadeau et al., "Multifrequency synthesis and extraction using square wave projection patterns for quantitative tissue imaging," J. Biomed. Opt. 20(11), 116005 (2015). 
62. M. Ghijsen et al., "Real-time simultaneous single snapshot of optical properties and blood flow using coherent spatial frequency domain imaging (cSFDI)," Biomed. Opt. Express 7(3), 870-882 (2016).

63. P. A. Valdes et al., "qF-SSOP: real-time optical property corrected fluorescence imaging," Biomed. Opt. Express 8(8), 3597-3605 (2017).

64. M. Xu et al., "Single snapshot multiple frequency modulated imaging of subsurface optical properties of turbid media with structured light," AIP Adv. 6(12), 125208 (2016).

65. M. Martinelli et al., "Analysis of single Monte Carlo methods for prediction of reflectance from turbid media," Opt. Express 19(20), 19627-19642 (2011).

66. O. Yang and B. Choi, "Accelerated rescaling of single Monte Carlo simulation runs with the graphics processing unit (GPU)," Biomed. Opt. Express 4(11), 2667-2672 (2013).

67. V. Pera et al., "Optical property uncertainty estimates for spatial frequency domain imaging," Biomed. Opt. Express 9(2), 661-678 (2018).

68. A. Liemert, D. Reitzle, and A. Kienle, "Analytical solutions of the radiative transport equation for turbid and fluorescent layered media," Sci. Rep. 7(1), 3819 (2017).

69. A. J. Lin et al., "Visible spatial frequency domain imaging with a digital light microprojector," J. Biomed. Opt. 18(9), 096007 (2013).

70. A. Darafsheh et al., "Performance characterization of a low-cost spatial frequency domain imaging system for the determination of optical properties in tissue-simulating phantoms and in vivo," Photodiagn. Photodyn. Ther. 12(3), 331-332 (2015).

71. R. H. Wilson et al., "Quantitative short-wave infrared multispectral imaging of in vivo tissue optical properties," J. Biomed. Opt. 19(8), 086011 (2014).

72. R. P. Singh-Moon et al., "Spatial mapping of drug delivery to brain tissue using hyperspectral spatial frequency-domain imaging," J. Biomed. Opt. 19(9), 096003 (2014).

73. M. Torabzadeh et al., "Compressed single pixel imaging in the spatial frequency domain," J. Biomed. Opt. 22(3), 030501 (2017).

74. A. M. Laughney et al., "System analysis of spatial frequency domain imaging for quantitative mapping of surgically resected breast tissues," J. Biomed. Opt. 18(3), 036012 (2013).

75. D. Cuccia, "Validation of spatial frequency domain imaging (SFDI) for biomedical research applications," in Imaging and Applied Optics 2015, p. AIT2C.2, OSA, Washington, D.C. (2015).

76. S. Tabassum et al., "Feasibility of spatial frequency domain imaging (SFDI) for optically characterizing a preclinical oncology model," Biomed. Opt. Express 7(10), 4154-4170 (2016).

77. J. Angelo, M. van de Giessen, and S. Gioux, "Real-time endoscopic optical properties imaging using single snapshot of optical properties (SSOP) imaging," Proc. SPIE 9313, 93130P (2015).

78. J. P. Angelo, M. van de Giessen, and S. Gioux, "Real-time endoscopic oxygenation imaging using single snapshot of optical properties (SSOP) imaging (conference presentation)," Proc. SPIE 9696, 969605 (2016).

79. N. Bodenschatz et al., "Diffuse optical microscopy for quantification of depth-dependent epithelial backscattering in the cervix," J. Biomed. Opt. 21(6), 066001 (2016).

80. J. Kress et al., "A dual-channel endoscope for quantitative imaging, monitoring, and triggering of doxorubicin release from liposomes in living mice," Sci. Rep. 7(1), 15578 (2017).

81. J. P. Angelo, M. van de Giessen, and S. Gioux, "Real-time endoscopic optical properties imaging," Biomed. Opt. Express 8(11), 5113-5126 (2017).

82. P. Ghassemi et al., "A polarized multispectral imaging system for quantitative assessment of hypertrophic scars," Biomed. Opt. Express 5(10), 3337-3354 (2014).

83. D. J. Rohrbach et al., "Preoperative mapping of nonmelanoma skin cancer using spatial frequency domain and ultrasound imaging," Acad. Radiol. 21(2), 263-270 (2014).

84. A. J. Lin et al., "Optical imaging in an Alzheimer's mouse model reveals amyloid- $\beta$-dependent vascular impairment," Neurophotonics 1(1), 011005 (2014).

85. D. M. McClatchy, III et al., "Calibration and analysis of a multimodal micro-CT and structured light imaging system for the evaluation of excised breast tissue," Phys. Med. Biol. 62(23), 8983-9000 (2017).

86. A. Mazhar et al., "Laser speckle imaging in the spatial frequency domain," Biomed. Opt. Express 2(6), 1553-1563 (2011).
87. T. B. Rice et al., "Determination of the effect of source intensity profile on speckle contrast using coherent spatial frequency domain imaging," Biomed. Opt. Express 3(6), 1340-1349 (2012).

88. T. B. Rice et al., "Quantitative, depth-resolved determination of particle motion using multi-exposure, spatial frequency domain laser speckle imaging," Biomed. Opt. Express 4(12), 2880-2892 (2013).

89. A. Ponticorvo et al., "Quantitative assessment of graded burn wounds in a porcine model using spatial frequency domain imaging (SFDI) and laser speckle imaging (LSI)," Biomed. Opt. Express 5(10), 3467-3481 (2014).

90. D. M. Burmeister et al., "Utility of spatial frequency domain imaging (SFDI) and laser speckle imaging (LSI) to non-invasively diagnose burn depth in a porcine model," Burns 41(6), 1242-1252 (2015).

91. A. Ponticorvo et al., "Quantitative long-term measurements of burns in a rat model using spatial frequency domain imaging (SFDI) and laser speckle imaging (LSI)," Lasers Surg. Med. 49(3), 293-304 (2017).

92. U. Sunar et al., "Quantification of PpIX concentration in basal cell carcinoma and squamous cell carcinoma models using spatial frequency domain imaging," Biomed. Opt. Express 4(7), 531-537 (2013).

93. B. Yang, M. Sharma, and J. W. Tunnell, "Attenuation-corrected fluorescence extraction for image-guided surgery in spatial frequency domain," J. Biomed. Opt. 18(8), 080503 (2013).

94. J. Kress et al., "Quantitative imaging of light-triggered doxorubicin release," Biomed. Opt. Express 6(9), 3546-3555 (2015).

95. M. Sibai et al., "Quantitative spatial frequency fluorescence imaging in the sub-diffusive domain for image-guided glioma resection," Biomed. Opt. Express 6(12), 4923-4933 (2015).

96. Y. Zhao, D. Roblyer, and A. J. Welch, "Spatial mapping of fluorophore quantum yield in diffusive media," J. Biomed. Opt. 20(8), 086013 (2015).

97. J. M. Schmitt, A. H. Gandjbakhche, and R. F. Bonner, "Use of polarized light to discriminate short-path photons in a multiply scattering medium," Appl. Opt. 31(30), 6535-6546 (1992).

98. V. Krishnaswamy et al., "Structured light scatteroscopy," J. Biomed. Opt. 19(7), 070504 (2014).

99. J. Angelo et al., "Depth-enhanced fluorescence imaging using masked detection of structured illumination," J. Biomed. Opt. 19(11), 116008 (2014).

100. J. Sun et al., "Enhancing in vivo tumor boundary delineation with structured illumination fluorescence molecular imaging and spatial gradient mapping," J. Biomed. Opt. 21(8), 080502 (2016).

101. B. Yang and J. W. Tunnell, "Real-time absorption reduced surface fluorescence imaging," J. Biomed. Opt. 19(9), 090505 (2014).

102. B. Yang et al., "Polarized light spatial frequency domain imaging for non-destructive quantification of soft tissue fibrous structures," Biomed. Opt. Express 6(7), 1520-1533 (2015).

103. B. Yang et al., "Color structured light imaging of skin," J. Biomed. Opt. 21(5), 050503 (2016).

104. S. Gioux et al., "First-in-human pilot study of a spatial frequency domain oxygenation imaging system," J. Biomed. Opt. 16(8), 086015 (2011).

105. A. Yafi et al., "Postoperative quantitative assessment of reconstructive tissue status in a cutaneous flap model using spatial frequency domain imaging," Plast. Reconstr. Surg. 127(1), 117-130 (2011).

106. A. Ponticorvo et al., "Quantitative assessment of partial vascular occlusions in a swine pedicle flap model using spatial frequency domain imaging," Biomed. Opt. Express 4(2), 298-306 (2013).

107. C. R. Vargas et al., "Intraoperative hemifacial composite flap perfusion assessment using spatial frequency domain imaging: a pilot study in preparation for facial transplantation," Ann. Plast. Surg. 76(2), 249255 (2016).

108. A. Ponticorvo et al., "Evaluating visual perception for assessing reconstructed flap health,” J. Surg. Res. 197(1), 210-217 (2015).

109. J. Q. Nguyen et al., "Spatial frequency domain imaging of burn wounds in a preclinical model of graded burn severity," J. Biomed. Opt. 18(6), 066010 (2013).

110. A. Mazhar et al., "Noncontact imaging of burn depth and extent in a porcine model using spatial frequency domain imaging," J. Biomed. Opt. 19(8), 086019 (2014).

111. T. T. A. Nguyen et al., "Novel application of a spatial frequency domain imaging system to determine signature spectral differences 
between infected and noninfected burn wounds," J. Burn Care Res. 34(1), 44-50 (2013).

112. A. J. Lin et al., "Spatial frequency domain imaging of intrinsic optical property contrast in a mouse model of Alzheimer's disease," Ann. Biomed. Eng. 39(7), 1349-1357 (2011).

113. A. J. Lin et al., "In vivo optical signatures of neuronal death in a mouse model of Alzheimer's disease," Lasers Surg. Med. 46(1), 27-33 (2014).

114. S. D. Konecky et al., "Spatial frequency domain tomography of protoporphyrin IX fluorescence in preclinical glioma models," J. Biomed. Opt. 17(5), 056008 (2012).

115. M. Sibai et al., "Preclinical evaluation of spatial frequency domainenabled wide-field quantitative imaging for enhanced glioma resection," J. Biomed. Opt. 22(7), 076007 (2017).

116. J. B. Travers et al., "Noninvasive mesoscopic imaging of actinic skin damage using spatial frequency domain imaging," Biomed. Opt. Express 8(6), 3045-3052 (2017).

117. A. M. Laughney et al., "Spectral discrimination of breast pathologies in situ using spatial frequency domain imaging," Breast Cancer Res. 15(7), R61 (2013).

118. S. Nandy et al., "Characterizing optical properties and spatial heterogeneity of human ovarian tissue using spatial frequency domain imaging," J. Biomed. Opt. 21(10), 101402 (2016).

119. J. Sharpe, "Optical projection tomography," Annu. Rev. Biomed. Eng. 6(1), 209-228 (2004).

120. M. S. Ozturk et al., "Mesoscopic fluorescence molecular tomography for evaluating engineered tissues," Ann. Biomed. Eng. 44(3), 667-679 (2016).

121. X. D. Li et al., "Fluorescence lifetime imaging in turbid media," Opt. Lett. 21(2), 158-160 (1996).

122. V. Ntziachristos et al., "In vivo tomographic imaging of near-infrared fluorescent probes," Mol. Imaging 1(2), 82-88 (2002).

123. J. Wu et al., "Fluorescence tomographic imaging in turbid media using early-arriving photons and Laplace transforms," Proc. Natl. Acad. Sci. U. S. A. 94(16), 8783-8788 (1997).

124. V. Ntziachristos and R. Weissleder, "Experimental three-dimensional fluorescence reconstruction of diffuse media by use of a normalized Born approximation," Opt. Lett. 26(12), 893-895 (2001).

125. S. Lam, F. Lesage, and X. Intes, "Time domain fluorescent diffuse optical tomography: analytical expressions," Opt. Express 13(7), 2263-2275 (2005).

126. B. W. Pogue et al., "Initial assessment of a simple system for frequency domain diffuse optical tomography," Phys. Med. Biol. 40(10), 17091729 (1995).

127. S. D. Konecky et al., "Imaging complex structures with diffuse light," Opt. Express 16(7), 5048-5060 (2008).

128. N. Ducros et al., "Fluorescence diffuse optical tomography: timeresolved versus continuous-wave in the reflectance configuration," IRBM 32(7), 243-250 (2011).

129. M. A. O'Leary et al., "Experimental images of heterogeneous turbid media by frequency-domain diffusing-photon tomography," Opt. Lett. 20, 426-428 (1995).

130. A. Godavarty, E. M. Sevick-Muraca, and M. J. Eppstein, "Threedimensional fluorescence lifetime tomography," Med. Phys. 32(7), 992-1000 (2005).

131. H. Zhao et al., "Imaging of in vitro chicken leg using time-resolved near-infrared optical tomography," Phys. Med. Biol. 47(11), 19791993 (2002).

132. A. T. Kumar et al., "Time resolved fluorescence tomography of turbid media based on lifetime contrast," Opt. Express 14(25), 12255-12270 (2006).

133. X. Intes and B. Chance, "Multi-frequency diffuse optical tomography," J. Mod. Opt. 52(15), 2139-2159 (2005).

134. J. Chen, V. Venugopal, and X. Intes, "Monte Carlo based method for fluorescence tomographic imaging with lifetime multiplexing using time gates," Biomed. Opt. Express 2(7), 871-886 (2011).

135. L. Hervé et al., "Time-domain diffuse optical tomography processing by using the Mellin-Laplace transform," Appl. Opt. 51(25), 59785988 (2012).

136. F. Gao et al., "Image reconstruction for tomographic mapping of cerebral hemodynamics using time-domain detection: simulation and phantom studies," in Photon Migration and Diffuse-Light Imaging, D. A. Boas, Ed., p. 148 (2003).
137. B. W. Pogue, M. S. Patterson, and T. J. Farrell, "Forward and inverse calculations for 3-D frequency-domain diffuse optical tomography," Proc. SPIE 2389, 328-339 (1995).

138. J. Chen, Q. Fang, and X. Intes, "Mesh-based Monte Carlo method in time-domain widefield fluorescence molecular tomography," J. Biomed. Opt. 17(10), 106009 (2012).

139. R. Yao, X. Intes, and Q. Fang, "Generalized mesh-based Monte Carlo for wide-field illumination and detection via mesh retessellation," Biomed. Opt. Express 7(1), 171-184 (2016).

140. A. H. Hielscher, "Optical tomographic imaging of small animals," Curr. Opin. Biotechnol. 16(1), 79-88 (2005).

141. X. Intes et al., "Interfering diffusive photon-density waves with an absorbing-fluorescent inhomogeneity," Opt. Express 8(3), 223-231 (2001).

142. Y. Chen et al., "Near-infrared phase cancellation instrument for fast and accurate localization of fluorescent heterogeneity," Rev. Sci. Instrum. 74(7), 3466-3473 (2003).

143. X. Intes, V. Ntziachristos, and B. Chance, "Analytical model for dual-interfering sources diffuse optical tomography," Opt. Express 10(1), 2-14 (2002).

144. J. Romberg, "Imaging via Compressive Sampling," IEEE Signal Process. Mag. 25(2), 14-20 (2008).

145. J. Chen et al., "Time-resolved diffuse optical tomography with patterned-light illumination and detection," Opt. Lett. 35(13), 21212123 (2010).

146. S. Bélanger et al., "Real-time diffuse optical tomography based on structured illumination," J. Biomed. Opt. 15(1), 016006 (2010).

147. V. Venugopal, J. Chen, and X. Intes, "Development of an optical imaging platform for functional imaging of small animals using wide-field excitation," Biomed. Opt. Express 1(1), 143-156 (2010).

148. V. Venugopal et al., "Quantitative tomographic imaging of intermolecular FRET in small animals," Biomed. Opt. Express 3(12), 3161-3175 (2012).

149. V. Lukic, V. A. Markel, and J. C. Schotland, "Optical tomography with structured illumination," Opt. Lett. 34(7), 983-985 (2009).

150. A. Joshi, W. Bangerth, and E. M. Sevick-Muraca, "Non-contact fluorescence optical tomography with scanning patterned illumination," Opt. Express 14(14), 6516 (2006).

151. J. Dutta et al., "Illumination pattern optimization for fluorescence tomography: theory and simulation studies," Phys. Med. Biol. 55(10), 2961-2982 (2010).

152. V. Venugopal et al., "Full-field time-resolved fluorescence tomography of small animals," Opt. Lett. 35(19), 3189-3191 (2010).

153. J. Chen and X. Intes, "Comparison of Monte Carlo methods for fluorescence molecular tomography-computational efficiency," Med. Phys. 38(10), 5788-5798 (2011).

154. L. Zhao et al., "Lp regularization for early gate fluorescence molecular tomography," Opt. Lett. 39(14), 4156-4159 (2014).

155. A. Bassi et al., "Novel approaches based on structured light for fast diffuse optical tomography," Proc. SPIE 7896, 78961T (2011).

156. D. A. Loginova et al., "Tissue sensing by structured illumination in optical diffuse reflectometry," Proc. SPIE 10336, 1033611 (2017).

157. N. Ducros et al., "A virtual source pattern method for fluorescence tomography with structured light," Phys. Med. Biol. 57(12), 38113832 (2012).

158. N. Ducros et al., "Full-wavelet approach for fluorescence diffuse optical tomography with structured illumination," Opt. Lett. 35(21), 3676-3678 (2010).

159. H. R. A. Basevi et al., "Simultaneous multiple view high resolution surface geometry acquisition using structured light and mirrors," Opt. Express 21(6), 7222-7239 (2013).

160. K. Baum et al., "3D-surface reconstruction method for diffuse optical tomography phantoms and tissues using structured and polarized light," Proc. SPIE 8088, 80881V (2011).

161. K. Baum et al., "Three-dimensional surface reconstruction within noncontact diffuse optical tomography using structured light," J. Biomed. Opt. 17(12), 126009 (2012).

162. R. Yao, Q. Pian, and X. Intes, "Wide-field fluorescence molecular tomography with compressive sensing based preconditioning," Biomed. Opt. Express 6(12), 4887-4898 (2015).

163. L. Zhao et al., "Active wide-field illumination for high-throughput fluorescence lifetime imaging," Opt. Lett. 38(19), 3976-3979 (2013). 
164. L. Zhao et al., "Spatial light modulator based active wide-field illumination for ex vivo and in vivo quantitative NIR FRET imaging," Biomed. Opt. Express 5(3), 944-960 (2014).

165. V. Venugopal and X. Intes, "Adaptive wide-field optical tomography," J Biomed Opt. 18(3), 036006 (2013).

166. N. Ducros et al., "Fluorescence molecular tomography of an animal model using structured light rotating view acquisition," J. Biomed. Opt. 18(2), 020503 (2013).

167. M. Pimpalkhare et al., "Ex vivo fluorescence molecular tomography of the spine," Int. J. Biomed. Imaging 2012, 1-11 (2012).

168. R. Yao, X. Intes, and Q. Fang, "A rapid approach to build Jacobians for optical tomography via Monte Carlo method and photon 'replay'," in Optics in the Life Sciences Congress, p. BoW3A.3, OSA, Washington, D.C. (2017).

169. A. Edmans and X. Intes, "Mesh optimization for Monte Carlo-based optical tomography," Photonics 2(7), 375-391 (2015).

170. M. Y. Berezin and S. Achilefu, "Fluorescence lifetime measurements and biological imaging," Chem. Rev. 110(5), 2641-2684 (2010).

171. M. D. Reisman et al., "Structured illumination diffuse optical tomography for noninvasive functional neuroimaging in mice," Neurophotonics 4(2), 021102 (2017).

172. K. Abe et al., "Non-invasive in vivo imaging of near infrared-labeled transferrin in breast cancer cells and tumors using fluorescence lifetime FRET," PLoS One 8(11), e80269 (2013).

173. S. Rajoria et al., "FLIM-FRET for cancer applications," Curr. Mol. Imaging 3(2), 144-161 (2015).

174. K. Abe et al., "Quantitative detection of near infrared-labeled transferrin using FRET fluorescence lifetime wide-field imaging in breast cancer cells in vitro and in vivo," in Imaging and Applied Optics, pp. 3-5, OSA, Washington, D.C. (2013).

175. V. Venugopal et al., "In vivo reconstruction of NIR FRET using fullfield time resolved optical tomography," Proc. SPIE 7892, 78920I (2011).

176. V. Venugopal and X. Intes, "Recent advances in optical mammography," Curr. Med. Imaging Rev. 8(3), 244-259 (2012).

177. M. F. Duarte et al., "Single-pixel imaging via compressive sampling," IEEE Signal Process. Mag. 25(2), 83-91 (2008).

178. S. S. Welsh et al., "Fast full-color computational imaging with singlepixel detectors," Opt. Express 21(20), 23068-23074 (2013).

179. F. Ferri et al., "High-resolution ghost image and ghost diffraction experiments with thermal light," Phys. Rev. Lett. 94(18), 183602 (2005).

180. Z. Zhang et al., "Hadamard single-pixel imaging versus Fourier singlepixel imaging," Opt. Express 25(16), 19619-19639 (2017).

181. F. Rousset et al., "Adaptive basis scan by wavelet prediction for singlepixel imaging," IEEE Trans. Comput. Imaging 3(1), 36-46 (2017).

182. J. Shin, B. T. Bosworth, and M. A. Foster, "Single-pixel imaging using compressed sensing and wavelength-dependent scattering," Opt. Lett. 41(5), 886 (2016).

183. Q. Pian et al., "Hyperspectral compressive single-pixel imager for fluorescence lifetime sensing," in Biomedical Optics, p. OTu2C.7, OSA, Washington, D.C. (2016).

184. B. Sun et al., "3D computational imaging with single-pixel detectors," Science 340(6134), 844-847 (2013).

185. M.-J. Sun et al., "Single-pixel three-dimensional imaging with timebased depth resolution," Nat. Commun. 7, 12010 (2016).

186. W. Becker, "Fluorescence lifetime imaging: techniques and applications," J. Microsc. 247(2), 119-136 (2012).

187. Q. Pian et al., "Compressive hyperspectral time-resolved wide-field fluorescence lifetime imaging," Nat. Photonics 11(7), 411-414 (2017).

188. B. Lochocki et al., "Single pixel camera ophthalmoscope," Optica 3(10), 1056-1059 (2016).

189. F. Soldevila et al., "High-resolution adaptive imaging with a single photodiode," Sci. Rep. 5(1), 14300 (2015).

190. G. Satat, M. Tancik, and R. Raskar, "Lensless imaging with compressive ultrafast sensing," IEEE Trans. Comput. Imaging 3(3), 398-407 (2017).

191. K. Guo, S. Jiang, and G. Zheng, "Multilayer fluorescence imaging on a single-pixel detector," Biomed. Opt. Express 7(7), 2425-2431 (2016).

192. G. Satat et al., "All photons imaging through volumetric scattering," Sci. Rep. 6(1), 33946 (2016)
193. E. Tajahuerce et al., "Image transmission through dynamic scattering media by single-pixel photodetection," Opt. Express 22(14), 1694516955 (2014).

194. N. Chakrova et al., "Studying different illumination patterns for resolution improvement in fluorescence microscopy," Opt. Express 23(24), 31367-31383 (2015).

195. Q. Pian et al., "Hyperspectral time-resolved wide-field fluorescence molecular tomography based on structured light and single-pixel detection," Opt. Lett. 40(3), 431-434 (2015).

196. E. J. Candès and T. Tao, "Near-optimal signal recovery from random projections: Universal encoding strategies?" IEEE Trans. Inf. Theory 52(12), 5406-5425 (2006).

197. E. J. Candès, "Compressive sampling," in Proc. of the Int. Congress, pp. 1433-1452 (2006).

198. D. L. Donoho, “Compressed sensing," IEEE Trans. Inf. Theory 52(7), 1289-1306 (2006).

199. L. Streeter et al., "Optical full Hadamard matrix multiplexing and noise effects," Appl. Opt. 48(11), 2078-2085 (2009).

200. R. Damaschini, "Limitation of the multiplex gain in Hadamard transform spectroscopy," Pure Appl. Opt. J. Eur. Opt. Soc. Part A 2(3), 173-177 (1993).

201. G. Nitzsche and R. Riesenberg, "Noise, fluctuation, and HADAMARD-transform spectrometry," Proc. SPIE 5111, 273-282 (2003).

202. M. G. L. Gustafsson, "Surpassing the lateral resolution limit by a factor of two using structured illumination microscopy," J. Microsc. 198(Pt. 2), 82-87 (2000).

203. D. S. Taubman, M. W. Marcellin, and M. Rabbani, JPEG2000: Image Compression Fundamentals, Standards and Practice, 2002 ed., Springer (2001).

204. S. Mallat, A Wavelet Tour of Signal Processing: the Sparse Way, 3rd ed., Academic Press (2008).

205. Q. S. Hanley, "Spectrally resolved fluorescent lifetime imaging," J. R. Soc. Interface 6(suppl. 1), S83-S92 (2009).

206. N. Radwell et al., "Single-pixel infrared and visible microscope," Optica 1(5), 285-289 (2014).

207. J. Peller, F. Farahi, and S. R. Trammell, "Single-pixel hyperspectral imaging for real-time cancer detection: detecting damage in ex vivo porcine tissue samples," Proc. SPIE 9791, 979100 (2016).

208. R. B. Sekar and A. Periasamy, "Fluorescence resonance energy transfer (FRET) microscopy imaging of live cell protein localizations," J. Cell Biol. 160(5), 629-633 (2003).

209. D. J. Rohrbach et al., "Characterization of nonmelanoma skin cancer for light therapy using spatial frequency domain imaging," Biomed. Opt. Express 6(5), 1761-1766 (2015).

210. J. T. Nguyen et al., "A novel pilot study using spatial frequency domain imaging to assess oxygenation of perforator flaps during reconstructive breast surgery," Ann. Plast. Surg. 71(3), 308-315 (2013).

211. N. Bodenschatz et al., "Dual-mode endomicroscopy for detection of epithelial dysplasia in the mouth: a descriptive pilot study," J. Biomed. Opt. 22(08), 1-10 (2017).

Joseph P. Angelo is a National Research Council fellow at the National Institute of Standards and Technology in the sensor science division of the Physical Measurement Laboratory. He obtained his $\mathrm{PhD}$ from Boston University in biomedical engineering, focusing on surgical guidance with real-time imaging technology. In his postdoctoral work, his research interests are to develop optical techniques for clinical imaging and material characterization.

Xavier Intes is a professor in the Department of Biomedical Engineering, codirector of the Biomedical Imaging Center at Rensselaer Polytechnic Institute, and an AIMBE fellow. He obtained his PhD from the Université de Bretagne Occidentale (France) and postdoctoral training at the University of Pennsylvania. He acted as the chief scientist of Advanced Research Technologies Inc., Montreal, Canada. His research interests are on the application of diffuse functional and molecular optical techniques for biomedical imaging in preclinical and clinical settings.

Biographies for the other authors are not available. 